\title{
Restoring mitofusin balance prevents axonal degeneration in a Charcot-Marie-Tooth type 2A model
}

\author{
Yueqin Zhou, ${ }^{1,2}$ Sharon Carmona, ${ }^{2}$ A.K.M.G. Muhammad, ${ }^{1,2}$ Shaughn Bell,, ${ }^{1,2}$ Jesse Landeros, ${ }^{1,2}$ Michael Vazquez, ${ }^{1,2}$ Ritchie Ho, ${ }^{2}$ \\ Antonietta Franco, ${ }^{3}$ Bin Lu, ${ }^{2}$ Gerald W. Dorn II, ${ }^{3}$ Shaomei Wang, ${ }^{2}$ Cathleen M. Lutz, ${ }^{4}$ and Robert H. Baloh ${ }^{1,2,5}$ \\ 'Center for Neural Science and Medicine, and ${ }^{2 B o a r d}$ of Covernors Regenerative Medicine Institute, Cedars-Sinai Medical Center, Los Angeles, California, USA. ${ }^{3}$ Center for Pharmacogenomics, Department of Internal \\ Medicine, Washington University School of Medicine, St. Louis, Missouri, USA. ${ }^{4}$ The Jackson Laboratory, Bar Harbor, Maine, USA. ${ }^{5}$ Department of Neurology, Cedars-Sinai Medical Center, Los Angeles, California, USA.
}

\begin{abstract}
Mitofusin-2 (MFN2) is a mitochondrial outer-membrane protein that plays a pivotal role in mitochondrial dynamics in most tissues, yet mutations in MFN2, which cause Charcot-Marie-Tooth disease type 2A (CMT2A), primarily affect the nervous system. We generated a transgenic mouse model of CMT2A that developed severe early onset vision loss and neurological deficits, axonal degeneration without cell body loss, and cytoplasmic and axonal accumulations of fragmented mitochondria. While mitochondrial aggregates were labeled for mitophagy, mutant MFN2 did not inhibit Parkin-mediated degradation, but instead had a dominant negative effect on mitochondrial fusion only when MFN1 was at low levels, as occurs in neurons. Finally, using a transgenic approach, we found that augmenting the level of MFN1 in the nervous system in vivo rescued all phenotypes in mutant $\mathrm{MFN2}^{\mathrm{R940}}$-expressing mice. These data demonstrate that the MFN1/MFN2 ratio is a key determinant of tissue specificity in CMT2A and indicate that augmentation of MFN1 in the nervous system is a viable therapeutic strategy for the disease.
\end{abstract}

\section{Introduction}

Mitofusin-2 (MFN2) is a mitochondrial GTPase that plays a pivotal role in mitochondrial dynamics, including regulation of mitochondrial fusion, transport, mitophagy, mtDNA stability, and mitochondria-ER interactions (1-5). Given the high-energy demand of neurons coupled with their unique cellular architecture (axons, dendrites), proper mitochondrial dynamics are required to provide energy when and where it is needed $(6,7)$. Disruption of mitochondrial dynamics has been linked to a diverse spectrum of neurological conditions, including Parkinson's disease and Alzheimer's disease $(7,8)$. In some of these cases, alterations in MFN2 function and mitochondrial dynamics may play a role in disease pathogenesis. For example, in Parkinson's disease, MFN2 plays a key role in PINK1-Parkin-mediated mitophagy $(5,9)$ and $M f n 2$ knockout in dopaminergic neurons impairs Parkin localization to mitochondria, leading to severe axonal loss (10). In Alzheimer's disease, MFN2 protein levels are decreased in both patient brain tissue and transgenic mouse models, and amyloid- $\beta$-mediated disruption of mitochondrial dynamics may contribute to the toxicity of amyloid- $\beta$ (11, 12). However, while MFN2 function has been implicated in a variety of diseases, MFN2 mutations are only directly responsible for Charcot-Marie-Tooth disease type 2A (CMT2A) (13).

\section{Related Commentary: p. 1533}

Conflict of interest: GWD is an inventor on patent application 15/710,696, submitted by Stanford University, which covers the use of peptide regulators of mitochondrial fusion in neurodegenerative disease.

Copyright: () 2019 American Society for Clinical Investigation

Submitted: August 14, 2018; Accepted: January 29, 2019.

Reference information: / Clin Invest. 2019;129(4):1756-1771.

https://doi.org/10.1172/JCl124194.
CMT is one of the most common inherited neurological disorders, and MFN2 mutations are the most frequently identified cause of axonal CMT (CMT type 2). Most cases are de novo dominant or show dominant inheritance, although recessive cases have also been reported $(13,14)$. The typical clinical features of CMT2A include early onset and progressive distal weakness, muscle atrophy, and sensory loss from degeneration of long peripheral axons (15). However, CMT2A represents a broader spectrum of neurologic disease, including optic atrophy (16), spastic paraparesis (17), hearing loss (18), developmental delay (19), and brain white matter changes (18). This indicates that, while long peripheral axons are most sensitive to MFN2 mutations, CMT2A is actually a broader disorder of primarily the central and peripheral nervous systems that can rarely also involve muscle or fat tissue $(20,21)$.

As MFN2 is ubiquitously expressed and loss of function is detrimental to many tissues $(1,4,10,22-25)$, it remains unclear what causes MFN2 mutations to preferentially affect the nervous system, although key candidates include the low level of MFN1 expression relative to other tissues and the unique energy demands of axons, which require proper mitochondrial transport and localization $(6,7,26)$. MFN1 can compensate for loss of MFN2 and protect the mitochondrial fusion and transport defects caused by MFN2 mutants in vitro $(26,27)$. However, it has been challenging to investigate the role of the MFN1/MFN2 ratio in vivo, as existing CMT2A transgenic mouse models have either a static congenital phenotype (28) or a mild late-onset phenotype (29-31), with none recapitulating the severity or broader spectrum of neurological damage observed in CMT2A patients.

To better understand the role of MFN2 mutation in vivo, we generated transgenic mice expressing either WT or point mutant $\left(\mathrm{MFN} 2^{\mathrm{R} 94 \mathrm{Q}}\right)$ under the neuronal-specific Thy1.2 promoter. Thy1.2-MFN2 ${ }^{R 94 Q}$ transgenic mice recapitulated a range 
A

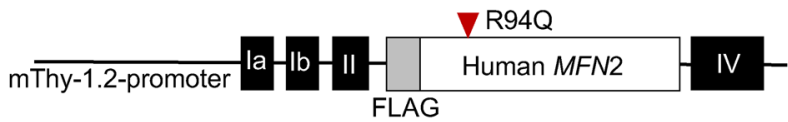

B

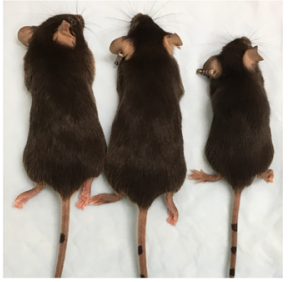

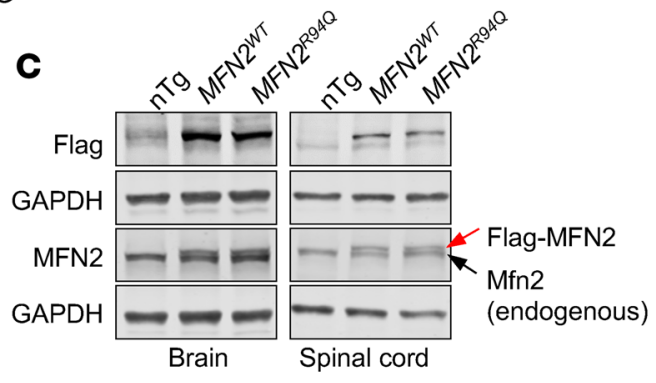

D

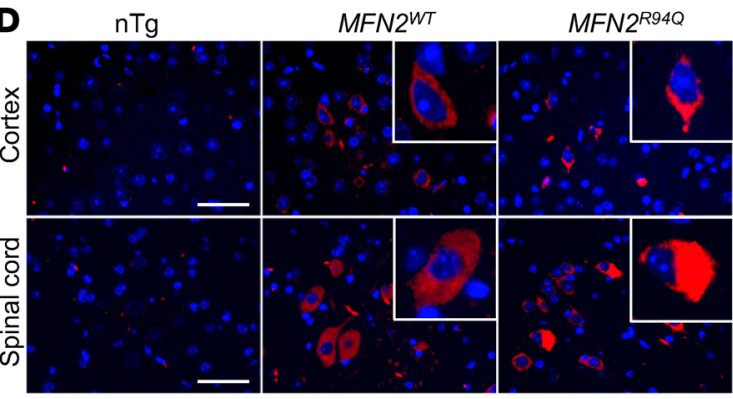

$\mathbf{F} \quad+\mathrm{nTg}$

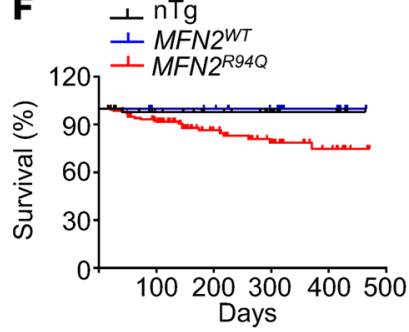

I
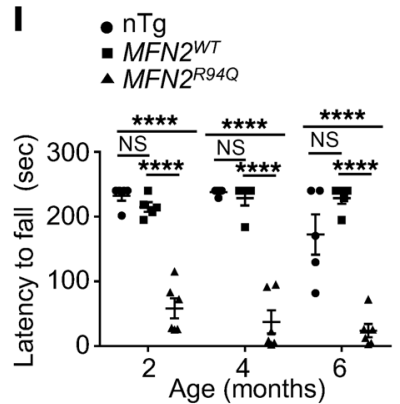

G $\quad$ onTg

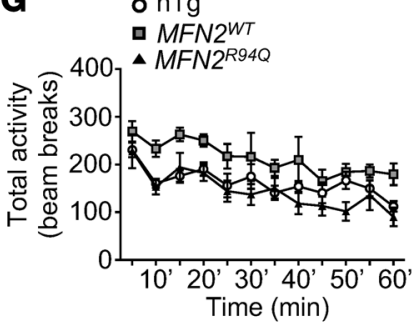

J

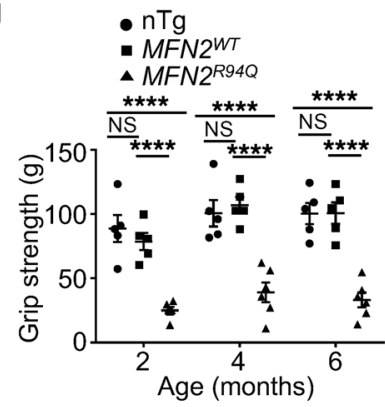

E

\section{o nTg}

- MFN2WT

- MFN2 ${ }^{R 94 Q}$
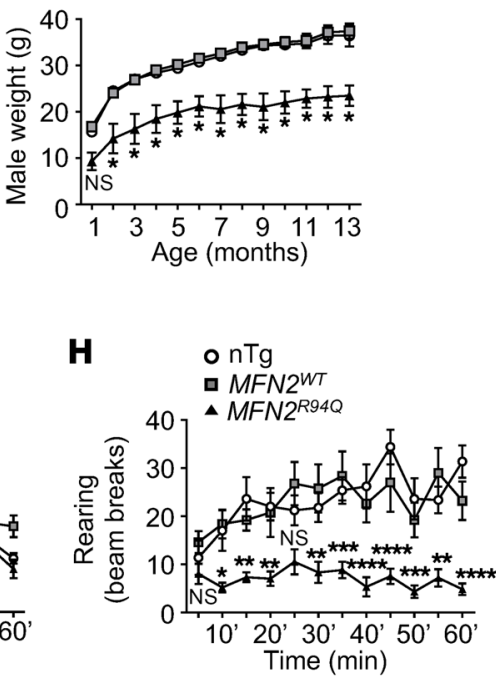

$\mathbf{K}$

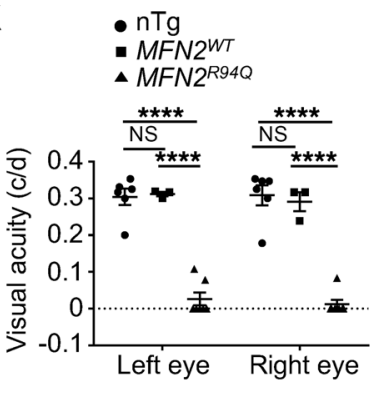

Figure 1. Impaired growth rate, sensorimotor function, grip strength, and vision in Thy1.2-MFN2 ${ }^{\text {R940 }}$ transgenic mice. (A) Schematic of the Thy1.2-MFN2 ${ }^{R 940}$ transgenic construct. The expression of human MFN2 ${ }^{\mathrm{Rg}}{ }^{9}$ or control MFN2 ${ }^{\mathrm{WT}}$ ( $\mathrm{N}$ terminus tagged with Flag) was driven by the neuron-specific mouse Thy1.2 promoter. (B) Representative image of a nontransgenic (nTg) mouse, a Thy1.2-MFN2 ${ }^{W T}$ mouse, and a Thy1.2-MFN2 ${ }^{\text {R940 }}$ mouse (5 months old). (C) Immunoblot of Flag-MFN2 ${ }^{\mathrm{WT}}$ or Flag-MFN2 ${ }^{\mathrm{R} 940}$ transgene expression (14-month-old mice). Red arrow, Flag-MFN2; black arrow, endogenous mouse Mfn2. Expression levels of MFN2 ${ }^{\mathrm{WT}}$ or MFN2 ${ }^{\mathrm{R} 940}$ transgenes were identical and slightly below endogenous Mfn2 levels. $n=3$ mice/genotype. (D) Immunostaining of Flag-MFN2 ${ }^{\text {WT }}$ or Flag-MFN2 ${ }^{\mathrm{R} 949}$ protein expression and localization. Mouse cortex or spinal cord (5-month-old mice). Anti-Flag (red) and DAPI (blue). Scale bars: $50 \mu \mathrm{M}$. Punctate mitochondrial staining was observed in both transgenic lines, but only MFN2 $2^{R 940}$ mice showed mitochondrial accumulations in neuronal cytoplasm and proximal axons. $n=3$ mice/genotype. (E) Body weight. Data are represented as mean \pm SEM. $n=6-52$ per genotype per time point. Student's 2-tailed $t$ test (nTg vs. MFN2 ${ }^{R 940}$ ). ${ }^{*} P<0.05$. (F) Survival

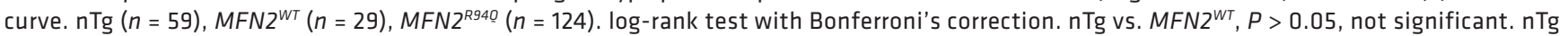
vs. MFN2 ${ }^{R 940}, P<0.01$. MFN2 ${ }^{W T}$ vs. MFN2 ${ }^{R 940}, P<0.05$. (G) Open-field test (total activity) and (H) open-field test (rearing). Total activity was not

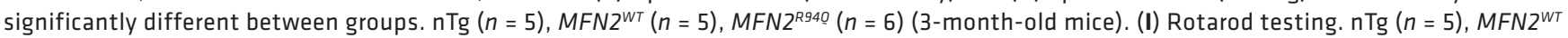

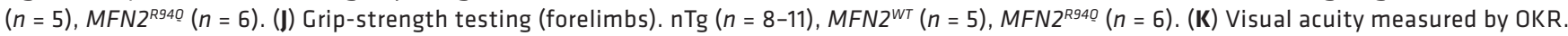
$\mathrm{nTg}(n=6)$, MFN2 $^{W T}(n=3)$, MFN2 $^{R 940}(n=7)$. In G-K, data are represented as mean \pm SEM. Two-way ANOVA with Tukey's test was used for multiple comparison. ${ }^{*} P<0.05 ;{ }^{* *} P<0.01 ;{ }^{* *} P<0.001 ;{ }^{* * *} P<0.0001$. 
of neurologic features seen in CMT2A patients, including severe early onset sensorimotor deficits, vision loss, altered mitochondrial dynamics, and widespread axonal degeneration. Increased expression of MFN1 in the nervous system rescued $M F N 2^{R 94 Q}$ mutant phenotypes, demonstrating that the balance of MFN isoforms is likely the key contributor to nervous system selectivity in CMT2A and providing proof of concept in vivo that augmenting MFN1 levels is a potential therapeutic strategy for the disease.

\section{Results}

Generation of transgenic mice expressing human $M F N 2^{W T}$ or $M F N 2^{R 94 Q}$ in neurons. We generated transgenic mice expressing Flag-tagged human MFN2 WT (MFN2 ${ }^{\mathrm{WT}}$ ) protein or mutant (MFN2 ${ }^{\mathrm{R} 94 \mathrm{Q}}$ ) under the Thy1.2 promoter to drive neuronal expression (Figure 1, A and B). We chose this promoter because it drives expression postnatally in most neurons, including projection neurons of the brain and spinal cord, which are most severely affected in CMT2A and show cell-autonomous defects from mutant MFN2 expression in vitro (32). We selected the R94Q variant, as dominant point mutations in this and neighboring residues in the GTPase domain have been reported across numerous ethnic backgrounds with a consistently severe phenotype with onset in the first decade that may include optic atrophy, spasticity, developmental delay, and brain white matter abnormalities $(13,17,33-35)$ and as previous in vitro studies demonstrated a dominant negative effect on mitochondrial fusion and transport $(3,26,27,32)$. We focused our analysis on 2 transgenic lines that showed comparable expression of either $\mathrm{MFN}^{\mathrm{WT}}$ (WT-47 line) or MFN2 ${ }^{\mathrm{R} 94 \mathrm{Q}}$ (R94Q-44 line). Because the Thy1.2-MFN2 ${ }^{R 94 Q}$ transgene was integrated into the Y chromosome in the R94Q-44 line, only male mice were analyzed for both lines. A second mutant transgenic line (R94Q-62) was also generated with a similar phenotype in both males and females (Supplemental Figure 1; supplemental material available online with this article; https://doi.org/10.1172/JCI124194DS1), but because it was more severe and difficult to propagate, we focused analysis on the R94Q-44 line.

Western blot using an antibody to Flag showed specific expression of transgenic MFN2 ${ }^{\mathrm{WT}}$ or $\mathrm{MFN} 2^{\mathrm{R} 94 \mathrm{Q}}$ protein at the expected size of approximately $84 \mathrm{kDa}$ (Figure 1C), with expression of the transgene slightly lower than that of endogenous mouse Mfn2 (Figure 1C). Immunofluorescence staining with anti-Flag antibody confirmed punctate cytoplasmic expression of exogenous MFN2 ${ }^{\mathrm{WT}}$ and $\mathrm{MFN} 2^{\mathrm{R} 94 \mathrm{Q}}$ that colocalized with COXIV, consistent with mitochondrial localization in neurons in brain and spinal cord (Figure 1D and Supplemental Figure 2). Occasionally, the center of the densest mitochondrial clusters showed diminished COXIV staining, which may represent partial degradation of mitochondrial components at the center of the cluster (Supplemental Figure 2). Of note, large accumulations of mitochondria were observed in neuronal cell bodies and proximal axons in cortex and spinal cord of Thy1.2-MFN2 ${ }^{R 94 Q}$ mice; these were not present in Thy1.2-MFN2 ${ }^{W T}$ mice (Figure 1D and Supplemental Figure 2). Similar mitochondrial clustering was also observed in the more severe R94Q-62 line (Supplemental Figure 1). These mitochondrial accumulations were reminiscent of those previously observed in cultured (DRG) neurons expressing MFN2 mutants $(28,32)$. The mutant $\mathrm{MFN} 2^{\mathrm{R} 94 \mathrm{Q}}$-induced mitochondrial accumulations were not due to MFN overexpression, as they were not seen in Thy1.2-MFN2 ${ }^{W T}$ transgenic mice.

Reduced growth rate, sensorimotor deficits, and vision loss in Thy1.2-MFN2 ${ }^{R 94 Q}$ mice. Both $M F N 2^{W T}$ and $M F N 2^{R 94 Q}$ mice were born at normal Mendelian ratios. While $M F N 2^{W T}$ mice were indistinguishable from nontransgenic mice, $M F N 2^{R 94 Q}$ mice showed stunted growth (Figure 1, B and E) and died prematurely from failure to thrive ( $25 \%$ died by 15 months of age; Figure $1 \mathrm{~F}$ ). By 3 months of age, $M F N 2^{R 94 Q}$ mice showed a wobbling gait defect and occasionally dragged their hind limbs (Supplemental Video 1). By 10 months, $M F N 2^{R 94 Q}$ mice had severe uncoordinated movements and hind limb paw clasping (Supplemental Figure 3), a neurologic finding commonly seen in mouse models of neurodegeneration (36).

No change in total activity was present on open-field testing; however, rearing activity (standing up on the side of the cage) was reduced in $M F N 2^{R 94 Q}$ mice (Figure $1, \mathrm{G}$ and $\mathrm{H}$ ). On rotarod testing, $M F N 2^{R 94 Q}$ mice fell off significantly earlier than either $M F N 2^{W T}$ or nontransgenic mice and did not improve over time (Figure 1I). $M F N 2^{R 94 Q}$ mice also showed weaker grip strength than $M F N 2^{W T}$ or nontransgenic control mice (Figure 1J). Given that some CMT2A patients have optic atrophy and vision loss, we examined response to visual stimulation on optokinetic response (OKR) testing and found that $M F N 2^{R 94 Q}$ mice also had severe vision loss (Figure $1 \mathrm{~K}$ ). While $M F N 2^{R 94 Q}$ mice declined in terms of overt coordination and clasping, most of these phenotypes were static and early onset. The early onset and sensorimotor deficits with vision loss in $M F N 2^{R 94 Q}$ mice are consistent with the broader spectrum of neurologic dysfunction seen in the more severe CMT2A patients, including those carrying the R94Q mutation who typically have onset in the first decade of life.

Axon atrophy and degeneration without neuronal cell body loss in $M F N 2^{\text {R94Q }}$ mice. CMT2A patients have a spectrum of neurologic dysfunction, including optic atrophy (16), spinal cord involvement with spastic paraparesis (17), and brain involvement with developmental delay $(18,19)$. We therefore examined $M F N 2^{R 94 Q}$ mice broadly for neuronal and axonal pathology in the central and peripheral nervous system. At 5 months of age, we observed diffuse axon degeneration on Fluoro-Jade staining in white matter tracts of the brain, including pyramidal tracts of the medulla and the lumbar spinal cord (Figure 2A). Surprisingly, in the distal tibial nerve, which typically shows severe axon loss in CMT2A patients, axons were smaller in caliber, but were normal in number, without degenerating profiles such as those seen in the spinal cord or brain (Figure 2, B and C). Similar axonal atrophy without degeneration has been observed in other mouse models of axonal CMT $(29,37,38)$, possibly due to shorter length, species differences, or lower promoter expression in peripheral neurons. In whole mounted retinas, neurofilament staining showed beaded, swollen, and degenerating axons with atrophic retinal ganglion cells (RGCs) in $M F N 2^{R 94 Q}$ mice (Figure 2D). In optic nerves, SMI32 staining showed significant neurofilament loss and axonal spheroids in $M F N 2^{R 94 Q}$ mice (Figure 2E and Supplemental Figure 4A), presumably explaining the vision loss in these animals. Interestingly, axon degeneration occurred in the absence of cell body loss throughout the nervous system, as no quantitative neuronal loss was observed in the retina, brain, or spinal cord, including motor cortex, sensory cortex, 
A

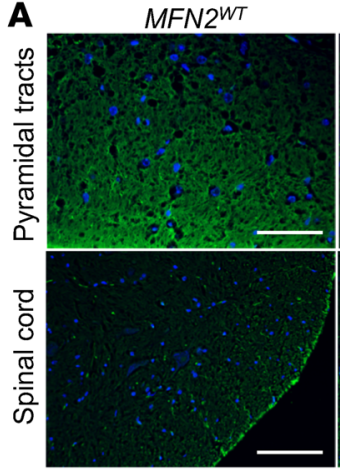

B

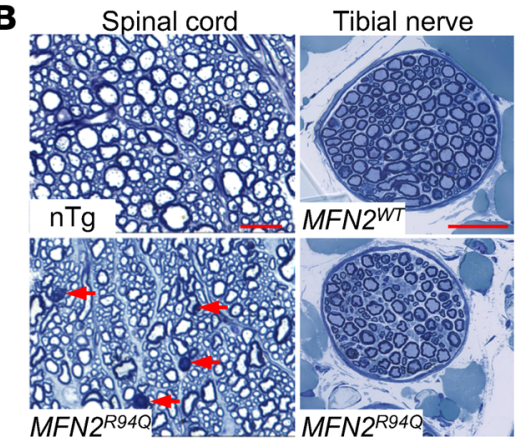

D
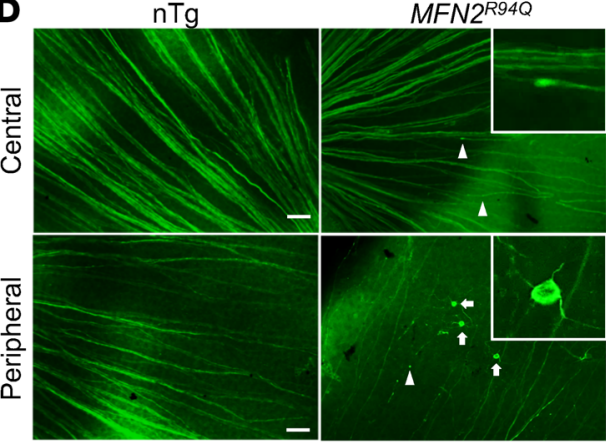

$\mathbf{F}$

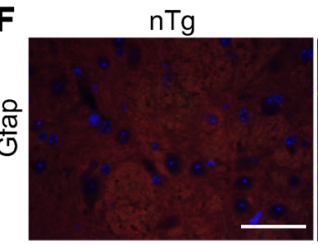

MFN2R94Q

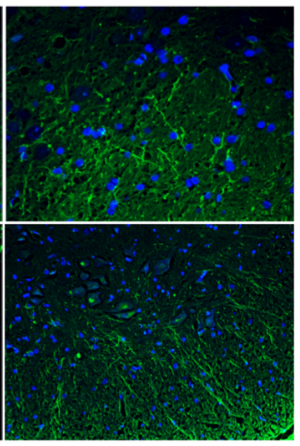

Tibial nerve

MFN2R94Q

MFN2R94Q

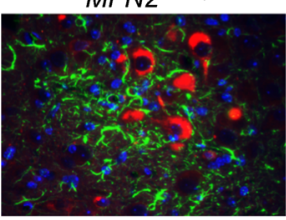

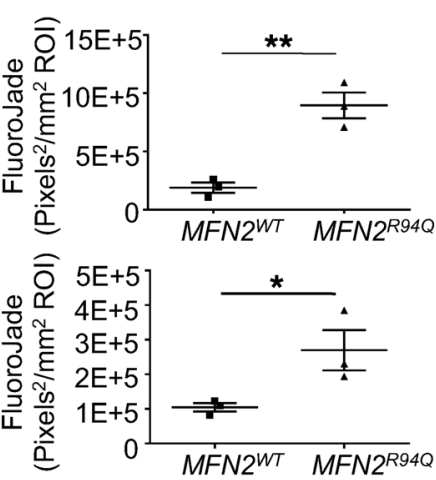

C

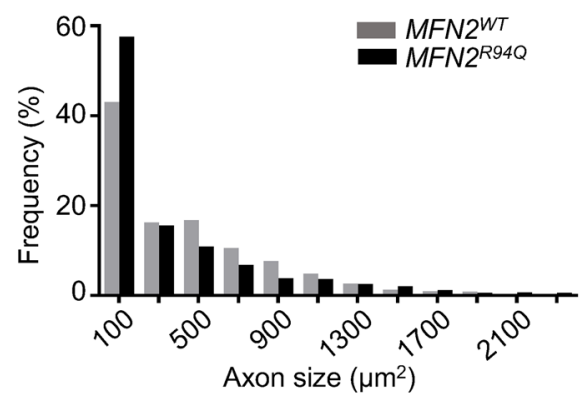

E
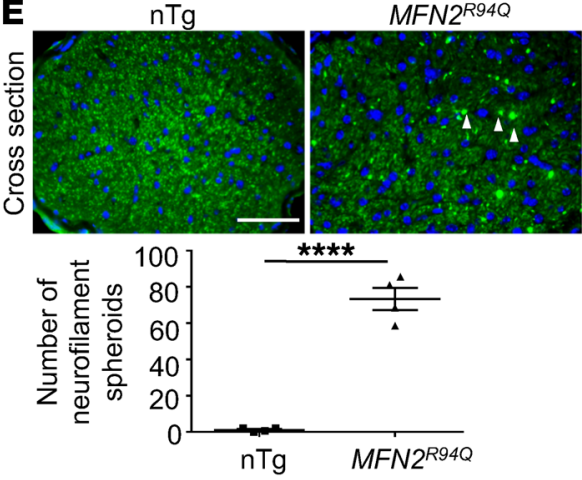

G
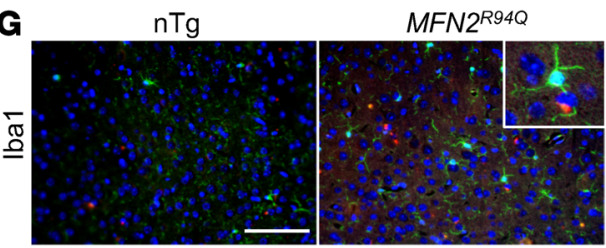

Figure 2. Widespread axon degeneration and gliosis without neuronal cell loss in MFN2 $\mathbf{R}^{\text {g40 }}$ mice. (A) Degenerating axons labeled with Fluoro-Jade staining in the brain pyramidal tracts of the medulla and the spinal cord of MFN2 ${ }^{R 940}$ mice (5 months old). Scale bar: $50 \mu \mathrm{M}$. Graph represents the quantification of Fluoro-Jade-positive signal as pixel $\mathrm{l}^{2}$ per $\mathrm{mm}^{2} \mathrm{ROI}$ subtracted from background. Data are represented as mean $\pm S E M$. $n=3$ mice/ genotype. Student's $t$ test, 2 -tailed for pyramidal track and 1-tailed for spinal cord. ${ }^{*} P<0.05$. (B) Toluidine blue-stained plastic sections of spinal cord and tibial nerves from 14-month-old nTg or MFN2 ${ }^{W T}$ and MFN2 ${ }^{R 940}$ mice. Scale bars: $10 \mu \mathrm{M}$ (spinal cord); $50 \mu \mathrm{M}$ (tibial nerve). Arrows indicate degenerating axons in the spinal cord. Tibial nerve showed axonal atrophy without axonal loss. $n=3$ mice/genotype. (C) Size frequency histogram of tibial nerve axons ( $n=3$ mice/group) showing axonal atrophy in MFN2 ${ }^{R 940}$ mice (Kolmogorov-Smirnov test, $P<0.0001$ ). (D) RGC axons stained with neurofilament antibody (RT97) in retinal whole-mount preparations. Scale bars: $50 \mu \mathrm{M}$. Arrowheads indicate beaded axons, and arrows indicate atrophic RGCs, seen only in MFN2 ${ }^{R 940}$ mice. $n=2$ mice/genotype. (E) Neurofilament in cross sections of optic nerve stained with SMI32 antibody. Scale bar: $50 \mu \mathrm{M}$. Axonal spheroids (white arrowheads) were present in the optic nerves of MFN2 ${ }^{\text {R94O }}$ mouse ( 3 months old). Graph represents the quantification of axonal spheroids. Data are represented as mean \pm SEM. $n=4$ /genotype. Student's $t$ test, 2 -tailed. ${ }^{* * *} P<0.0001$. (F) Astrocyte activation was present in regions of $M F N 2^{R 940}$ mouse brains (brain stem shown here) in areas of projection neurons that contained mitochondrial aggregates (5-month-old mice). GFAP (green); Flag (red); DAPI (blue). Scale bar: $50 \mu \mathrm{M} . n=3$ mice/genotype. (G) Microgliosis in regions of MFN2 ${ }^{R 940}$ mouse brains (cortex shown here) (5-month-old mice). Iba1 (green); Flag (red); DAPI (blue). Scale bar: $100 \mu \mathrm{M} . n=3$ mice/genotype. 
A

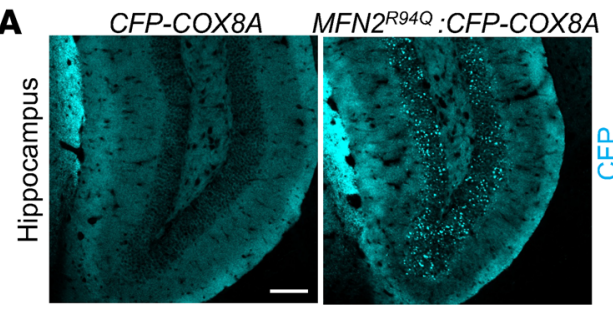

C
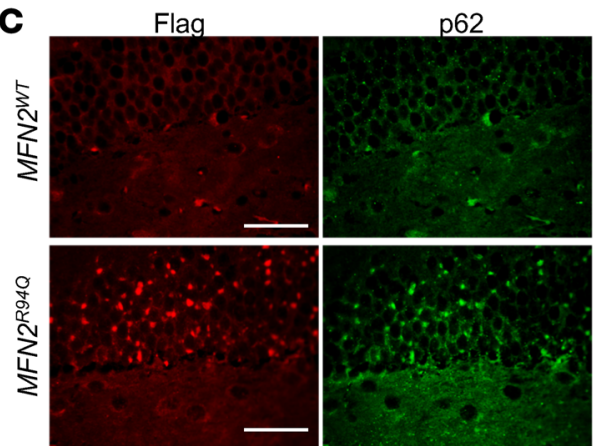

D
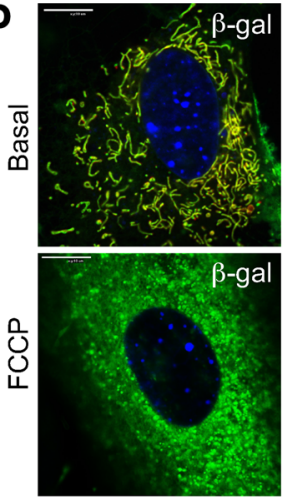

$\mathbf{F}$

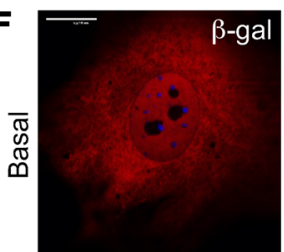

언
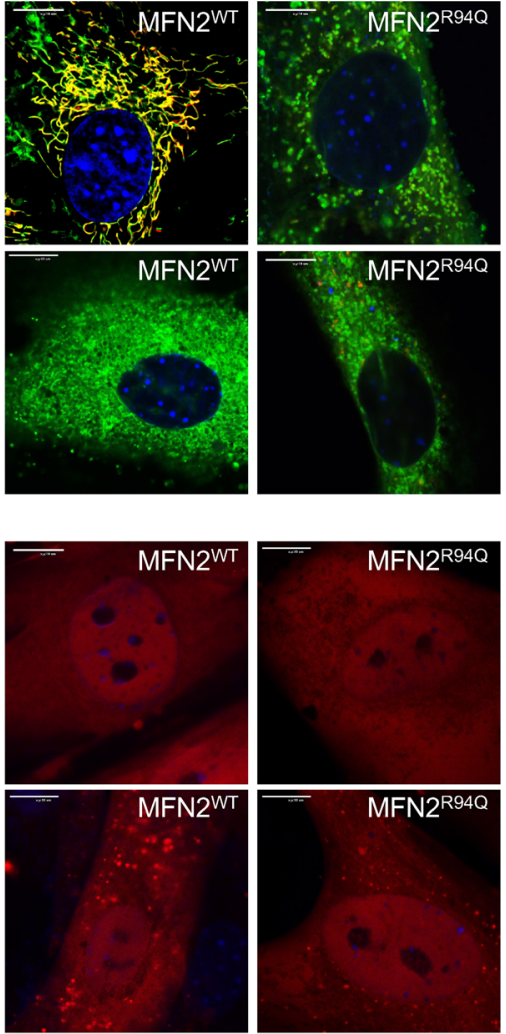

H
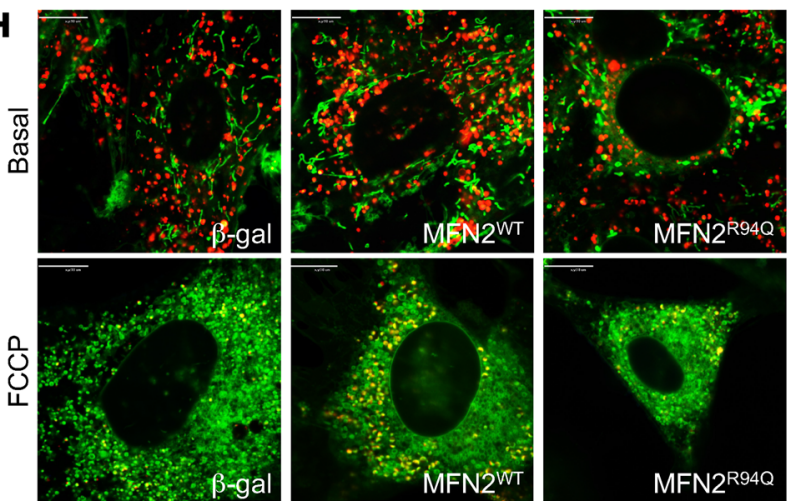

overlay
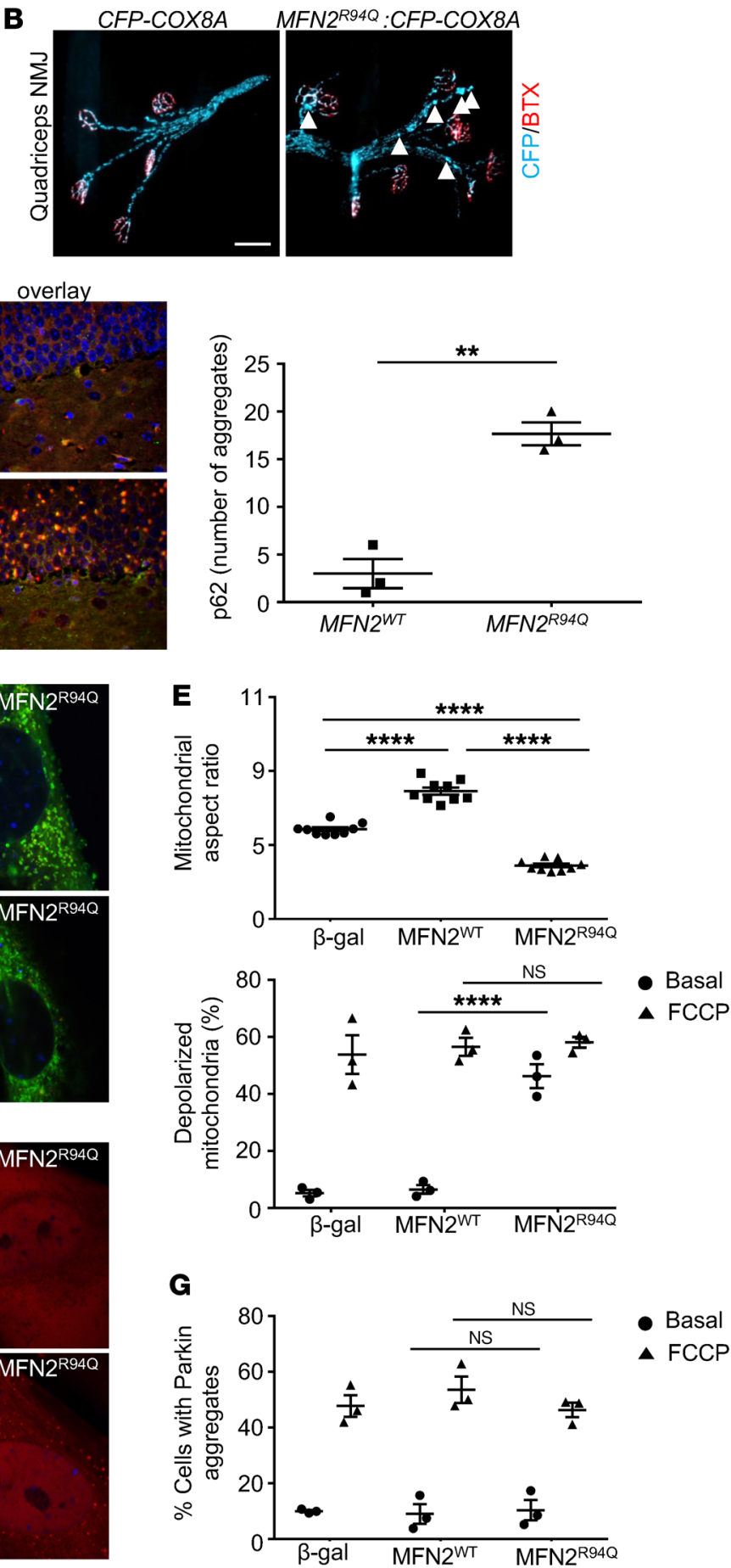

I

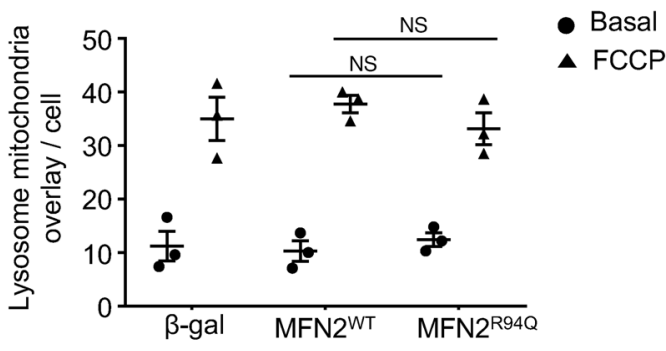


Figure 3. Dominant negative disruption of mitochondrial fusion, but not mitophagy, by MFN2 ${ }^{\mathrm{R} 94}$. (A) Cytoplasmic accumulations of mitochondria in the hippocampus of $M F N 2^{R 940}$ :CFP-COX8A mutant mice, not seen in CFP-COX8A control mice (7-month-old mice). Scale bar: 100 $\mu \mathrm{M} . n=2$ mice/genotype. (B) Mitochondrial clustering was also seen in distal peripheral nerve and NMJ of MFN2 ${ }^{R 940}$ :CFP-COX8A mice (quadriceps muscle shown). NMJ was labeled with $\alpha$-bungarotoxin (BTX, red). Arrowheads indicate mitochondrial clusters in distal axons (7-month-old mice). Scale bar: $50 \mu$ M. $n=2$ mice/genotype. (C) Mitochondrial accumulations in MFN2 ${ }^{R 940}$ mice were colocalized with p62 protein, indicating they were marked for mitophagy. Anti-Flag staining labels transgenic MFN2. DAPI labels nuclei (5-month-old mice). Scale bars: $50 \mu \mathrm{M}$. Numbers of p62 aggregates per ROI $\left(0.01 \mathrm{~mm}^{2}\right)$ are quantified. Data are represented as mean \pm SEM. $n=3$ mice/genotype. Student's 2-tailed $t$ test, ${ }^{* *} P<0.01$. (D) Effects of MFN2 ${ }^{\mathrm{R} 949}$ on mitochondrial fusion, membrane potential, and mitophagy in MEFs. MEFs were infected with $\beta$-gal (control), MFN2 ${ }^{\mathrm{WT}}$, or MFN2 ${ }^{\mathrm{R} 940}$ lentivirus. Depolarization was induced by treating MEFs with FCCP $(10 \mu \mathrm{M})$ for 1 hour. Mitochondria were labeled with MitoTracker (green) and TMRE (red). Scale bar: $10 \mu \mathrm{M}$. (E) Quantification of $\mathbf{D}$. Mitochondrial fusion at the basal level is presented as mitochondrial aspect ratio measurement (mitochondrial length/width; $n=9$ repeat experiments). Initiation of mitophagy is presented as mitochondrial depolarization ratio (green/green+yellow mitochondria; $n=3$ ). (F) Parkin localization. MEFs were infected with adenovirus expressing RFP-parkin. Scale bars: $10 \mu \mathrm{M}$. (G) Quantification of parkin localization in $\mathbf{F}(n=3$ repeat experiments). (H) Colocalization of mitochondria and lysosome. Mitochondria were labeled with MitoTracker Green, and lysosomes were labeled with LysoTracker Red. Scale bars: $10 \mu \mathrm{M}$. (I) Quantification of mitochondria and lysosome colocalization in $\mathbf{H}(n=3$ repeat experiments). In E, G, and $\mathbf{I}$, data are represented as mean $\pm \mathrm{SEM}$. One-way ANOVA with Tukey's test. ${ }^{* *} P<0.01 ;{ }^{* * *} P<0.0001$.

and ventral horn (Supplemental Figure 4, B and C, and Supplemental Figure 5A). While no neuronal degeneration was observed, reactive astro- and microgliosis (enhanced Gfap and Iba1 immunoreactivity) were observed adjacent to projection neurons in cortical layer 5 and brain stem motor nuclei (Figure 2, F and G, and Supplemental Figure 5, B and C). Costaining with Flag to label mutant MFN2 ${ }^{\mathrm{R} 94 \mathrm{Q}}$ containing mitochondria showed that gliosis was most pronounced adjacent to neurons with cytoplasmic accumulations of mitochondria (Figure 2F). These results indicate that $\mathrm{MFN} 2^{\mathrm{R} 94 \mathrm{Q}}$ expression produced widespread axon degeneration in the brain and spinal cord, particularly in regions containing long projection neurons. While neuronal cell bodies showed cytoplasmic mitochondrial accumulations and adjacent gliosis, no cell body loss was observed.

Mitochondrial clustering and morphology abnormalities induced by $M F N 2^{R 94 Q}$. To better visualize mitochondrial morphology and accumulations, we crossed $M F N 2^{R 94 Q}$ mice to transgenic mice with cyan fluorescent protein-labeled (CFP-labeled) mitochondria (39). By 8 months, accumulations of CFP-labeled mitochondria were observed in hippocampus, cortex, thalamus, cerebellum, brain stem, and spinal cord in $M F N 2^{R 94 Q}: C F P-C O X 8 A$ double-transgenic mice (Figure $3 \mathrm{~A}$ and Supplemental Figure 6A); these were similar to what was seen with anti-Flag antibody (Figure 1D). Despite the accumulation of mitochondria in spinal motor neurons, mitochondria were present in distal axons and at neuromuscular junctions (NMJs), although they were frequently clumped or clustered (Figure 3B); this is reminiscent of findings seen in nerve biopsy samples from patients with CMT2A $(40,41)$. While NMJs showed abnormal shape in the $M F N$ $2^{\text {R94Q }}$ CFP-COX8A double-transgenic mice, NMJ occupancy was normal at 8 months of age (Figure $3 \mathrm{~B}$ ). Neuronal cytoplasmic mito- chondrial accumulations were positive for both p62 and ubiquitin (Figure 3C and Supplemental Figure 6, B-E), indicating they were marked for degradation by mitophagy. While early studies reported mitochondrial clustering from extremely high-level WT MFN2 overexpression in tissue culture (42), no mitochondrial clustering was observed in $M F N 2^{W T}$ transgenic mice despite similar levels of expression, indicating this was a mutant-specific effect (Figure 3C).

Dominant negative disruption of mitochondrial fusion, but not mitophagy, by $M F N 2^{R 94 Q}$. Accumulations of fragmented mitochondria in cell bodies and axons from CMT2A-related MFN2 mutants were previously demonstrated in cultured neurons, together with disruption of mitochondrial fusion and transport induced by $\mathrm{MFN} 2^{\mathrm{R} 94 \mathrm{Q}}$ $(3,26,32)$. However, given subsequent studies defining a role of MFN2 in mitophagy $(5,9)$, we investigated whether mutant MFN2 ${ }^{\mathrm{R} 94 \mathrm{C}}$ could disrupt mitophagy directly, leading to accumulation of mitochondria in the cell body that could not be degraded. Therefore, we examined mouse embryonic fibroblasts (MEFs) expressing $\mathrm{MFN} 2^{\mathrm{WT}}$ or $\mathrm{MFN} 2^{\mathrm{R} 94 \mathrm{Q}}$ via lentiviruses. The mitochondrial aspect ratio (length/width), a measure of mitochondrial fusion (43), was significantlydecreased in MEFs expressing MFN2 ${ }^{\mathrm{R} 94 \mathrm{Q}}$, butincreased in MEFs expressing MFN2 ${ }^{\mathrm{WT}}$, in comparison with control MEFs (Figure 3, D and E). Additionally, a significant $(P<0.0001)$ increase in depolarized mitochondria was observed in MEFs expressing MFN2 ${ }^{\mathrm{R} 94 \mathrm{Q}}$ compared with control MEFs or those expressing $\mathrm{MFN}^{\mathrm{WT}}$ (Figure 3, D and E). These data indicate that MFN2 ${ }^{\mathrm{R} 94 \mathrm{Q}}$ suppresses mitochondrial fusion in a dominant negative manner and that this suppression is accompanied by disruption of mitochondrial membrane potential. To assess mitophagy directly, we treated MEFs with trifluoromethoxy carbonyl cyanide phenylhydrazone (FCCP), which uncouples oxidative phosphorylation and ATP production and leads to Parkin-dependent mitophagy (5). Parkin translocation from the cytosol to mitochondria was not different between MEFs expressing MFN2 ${ }^{\mathrm{R} 94 \mathrm{Q}}$ and controls (Figure $3, \mathrm{~F}$ and $\mathrm{G}$ ), indicating $\mathrm{MFN} 2^{\mathrm{R} 94 \mathrm{Q}}$ is recognized normally by the Pink1/Parkin mitophagy pathway. Additionally, mitochondrialysosome colocalization was normal in MFN2 ${ }^{\mathrm{R} 94} \mathrm{Q}$-expressing MEFs and increased similarly after mitophagy induction with FCCP treatment (Figure 3, H and I). These data indicate that mutant MFN2 ${ }^{\mathrm{R} 94 \mathrm{Q}}$ does not disrupt Parkin-mediated mitophagy and that the mitochondrial accumulations observed in $M F N 2^{R 94 Q}$ mice are not due to a direct block in mitochondrial degradation.

Conformation-altering peptides and MFN1 complementation for MFN2 ${ }^{R 94 Q}$-induced defects. The $M F N 2^{R 94 Q}$ mutation lies adjacent to the GTPase domain, which is a hot spot for CMT2A mutations (44). While there are several models of MFN2 structure and conformation during mitochondrial fusion, all require GTPase function for fusion of the outer mitochondrial membrane (43, 45-48). In the most well-studied model, initial tethering involves C-terminal heptad-repeat 2 (HR2) domains in trans, with subsequent GTPase domain-mediated GTP hydrolysis $(43,45)$. Therefore, MFN2 GTPase mutants such as $\mathrm{MFN} 2^{\mathrm{R} 94 \mathrm{Q}}$ are competent for initial tethering with MFN1 or MFN2 on adjacent mitochondria, but are unable to complete fusion (Figure 4A).

To test this model and investigate potential therapeutic strategies for CMT2A, we first examined the effect of a recently described peptide (TAT-MP1 ${ }^{\text {Gly }}$ ) that allosterically enhances mitochondrial fusion through altering C-terminal heptad repeat (HR1-HR2) interactions 
A
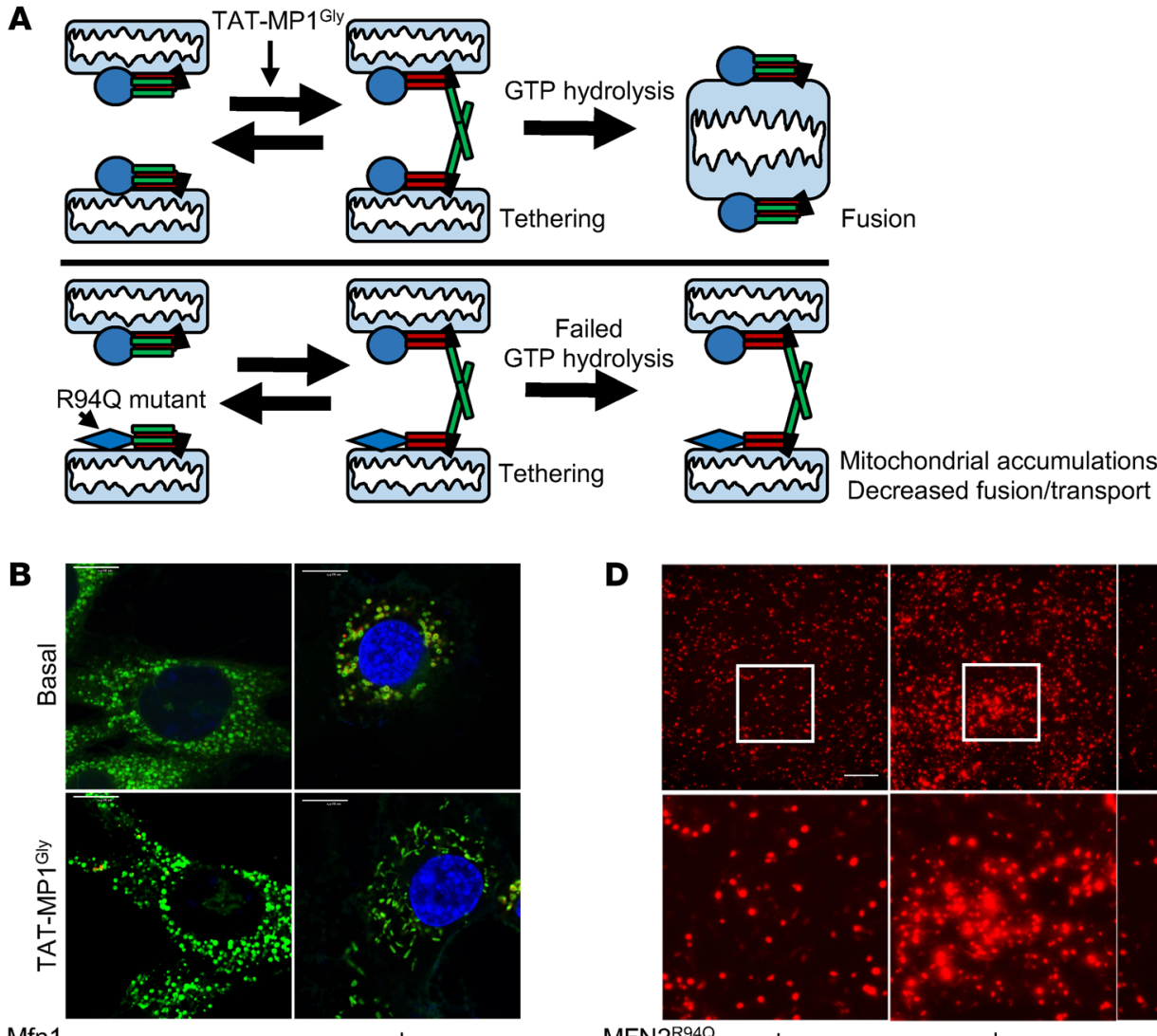

D
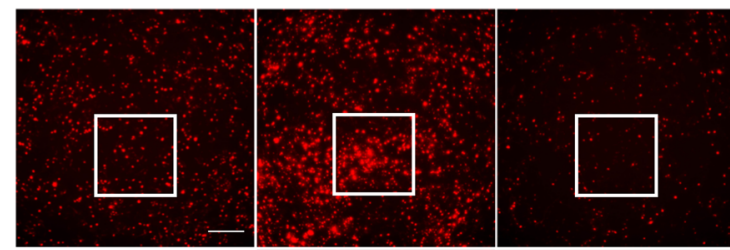

Mfn2

MFN2 $294 \mathrm{Q}+$
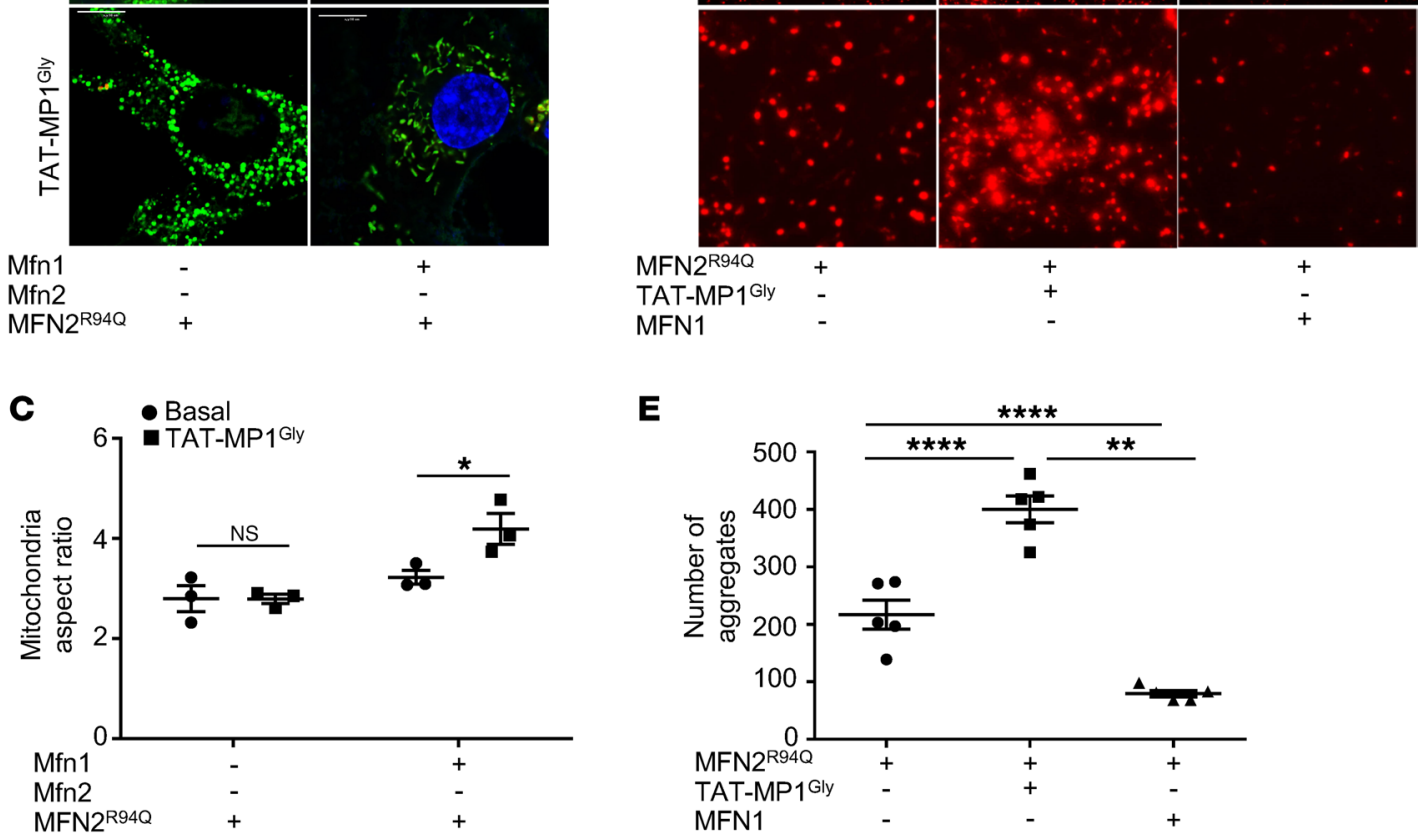

$\mathbf{E}$

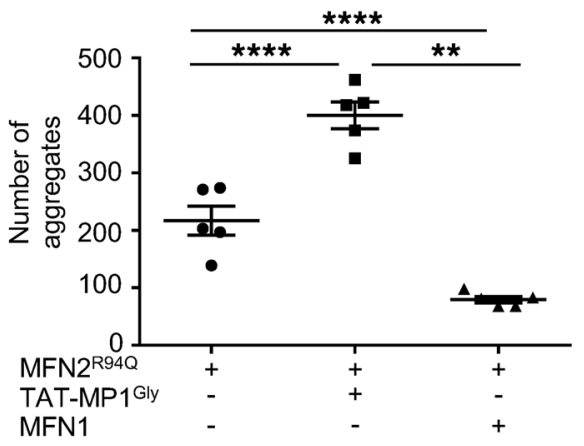

Figure 4. Model of dominant negative function by MFN2 ${ }^{\mathrm{R} 949}$ mutation and effect of conformation-altering peptides and MFN1 complementation. (A) Model of MFN2 ${ }^{\mathrm{Rg} 40}$ mutant dominant negative function and effect of allosteric peptide TAT-MP1 ${ }^{\mathrm{Cly}}$. Alteration of GTPase activity does not affect initial mitochondrial tethering (mediated by HR2 domain), but leads to inability to fuse after tethering. TAT-MP1'ly peptide drives the tethering permissive state in this model by blocking HR1-HR2 interaction. (B) Mitochondrial fusion defects caused by MFN2 ${ }^{\mathrm{R} 4 \mathrm{Q}}$ are only rescued by TAT-MP1 ${ }^{\mathrm{Cly}}$ in the presence of Mfn1 in MEFs. MEFs lacking either Mfn2 alone or both Mfn1 and Mfn2 were infected with MFN2 ${ }^{\mathrm{R} 940}$ lentivirus and imaged with MitoTracker Green and TMRE to label mitochondria. Scale bar: $10 \mu \mathrm{M}$. (C) Quantification of B. Mitochondrial fusion is presented as mitochondrial aspect ratio measurement (mitochondrial length/width). TAT-MP1 ${ }^{\text {Cly }}$ only improved mitochondrial fusion in the presence of functioning Mfn1, but not in cells lacking all functional Mfns. Data are represented as mean \pm SEM ( $n=3$ repeat experiments). Student's 2-tailed $t$ test. ${ }^{*} P<0.05$. (D) MFN2 ${ }^{\text {R940-induced }}$ mitochondrial aggregation was enhanced by TAT-MP1 ${ }^{\text {Cly }}$ peptide in a neuronal cell line (SH-SY5Y cells) and rescued by increased MFN1 expression. MFNs were expressed by lentivirus, with mito-RFP to visualize mitochondria. Original magnification, $\times 20$ (upper panels). Inset boxes are shown at higher magnification below ( $\times 3$ magnification). Scale bar: $100 \mu \mathrm{M}$. Representative of 3 repeat experiments. (E) Quantification of $\mathbf{D}$. Data are represented as mean \pm SEM $(n=5)$. One-way ANOVA with Tukey's test was used for multiple comparisons. ${ }^{* *} P<0.01 ;{ }^{* * *} P<0.0001$.

and promoting a "tethering permissive" state (43). We observed that the TAT-MP1 ${ }^{\text {Gly }}$ peptide partially rescued the fusion defect caused by $\mathrm{MFN} 2^{\mathrm{R} 94 \mathrm{Q}}$ in MEFs lacking endogenous mouse Mfn2 (but retaining Mfn1), but could not rescue fusion in double-knockout MEFs (lacking both Mfn1 and Mfn2) (Figure 4, B and C). This supports that MFN2 ${ }^{\mathrm{R} 94 \mathrm{Q}}$ by itself is fusion incompetent and that the TAT-MP1 ${ }^{\text {Gly }}$ peptide requires at least one functioning Mfn isoform (in this case Mfn1) to promote fusion as well as that presumably in MEFs, the peptide is 
A
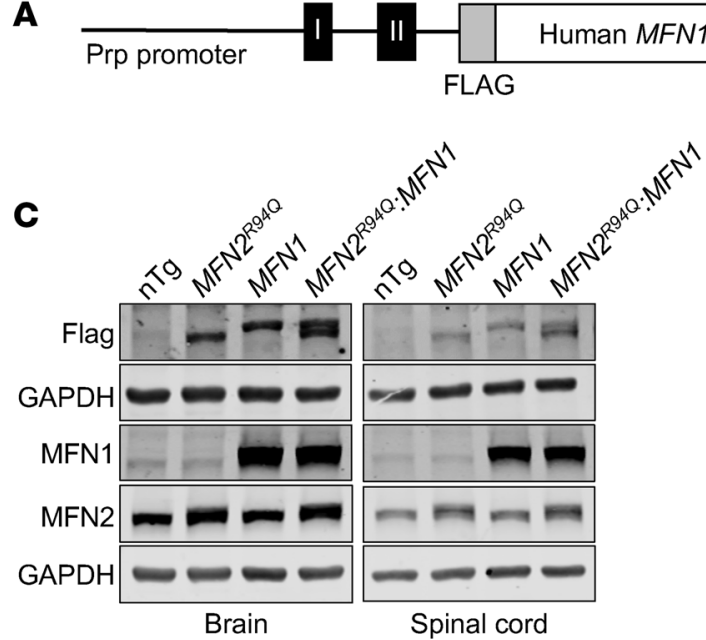

III Human MFN1

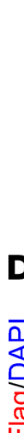

D
B
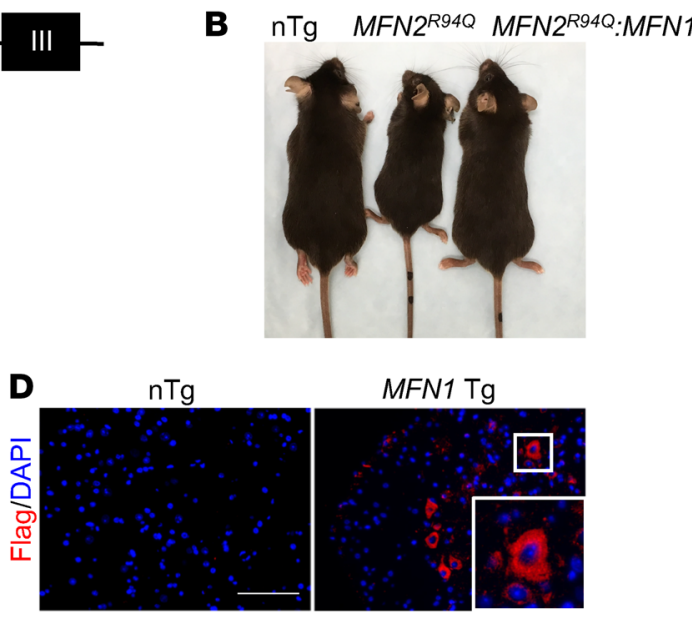

MFN1 Tg
E

$$
\begin{aligned}
& \text { - }{ }^{\mathrm{nTg}} \\
& \text { ล } \text { MFN2R94Q }^{\text {O }} \text { MFN2R94Q:MFN1 }
\end{aligned}
$$

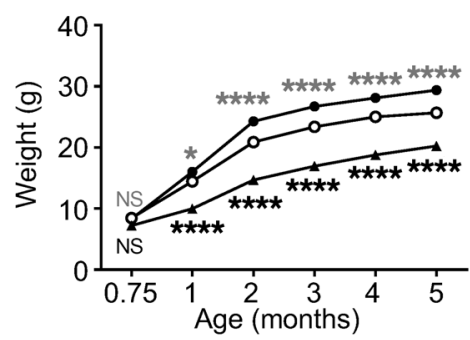

H
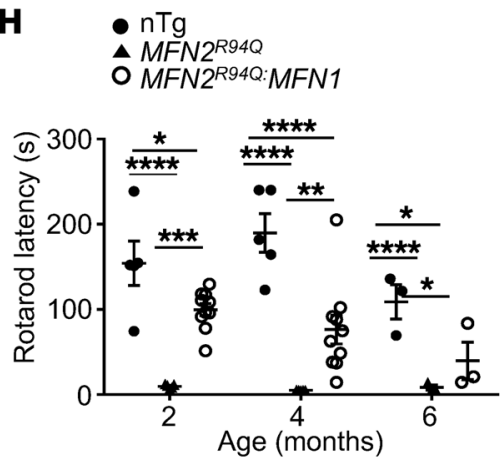

F $\quad \perp \mathrm{nTg}$

$\perp$ MFN2R94Q

I MFN2 ${ }^{\text {R94Q:MFN1 }}$

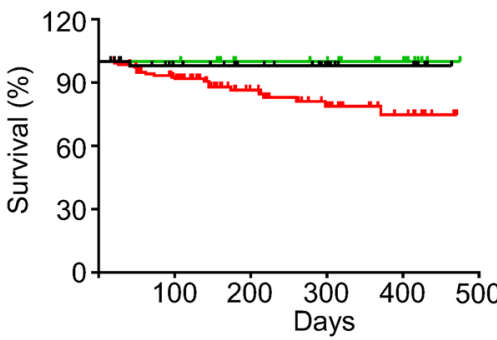

I $\mathrm{nTg}$

- MFN2R94Q

O MFN2 ${ }^{\text {R94Q:MFN1 }}$

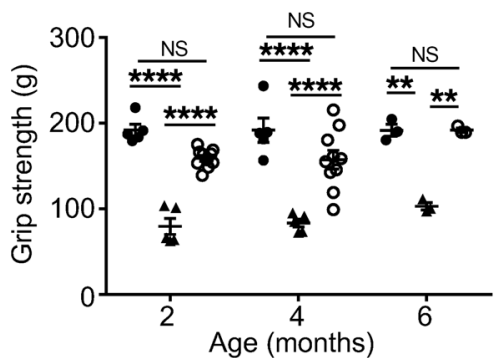

G $\quad \mathrm{nTg}$

- MFN2R94Q

O MFN2 ${ }^{R 94 Q: M F N 1}$

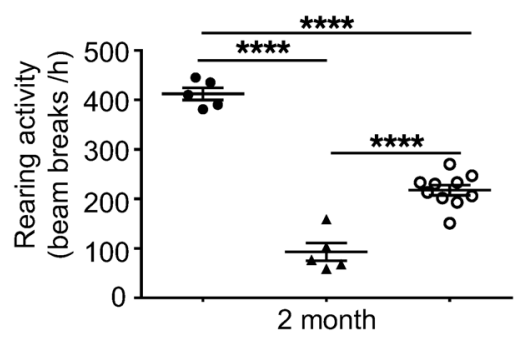

J

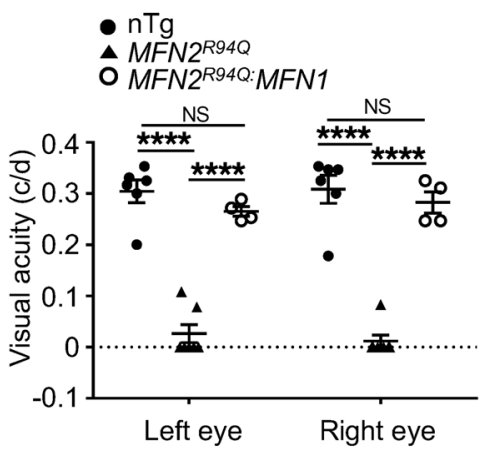

Figure 5. Phenotypic rescue of $M F N 2^{R 940}$ mice by augmenting MFN1 expression in the nervous system. (A) Schematic of the Prp-MFN1 transgene. The expression of human MFN1 (N terminus tagged with Flag) in brain and spinal cord is driven by the murine Prp gene promoter. Prp-MFN1 transgenic mice were crossed with Thy1.2-MFN2 ${ }^{\text {R940 }}$ transgenic mice to generate Thy1.2-MFN2 ${ }^{R 940}$ :Prp-MFN1 double-transgenic mice. (B) Representative image of a 20-week-old MFN2 ${ }^{R 940}:$ MFN1 double-transgenic mouse compared with a MFN2 ${ }^{R 940}$ mutant mouse and an nTg control. (C) MFN1 and/ or MFN2 ${ }^{\mathrm{R} 94}$ transgene expression detected by immunoblotting. Lysates of 14 -month-old mouse brain and spinal cord. Expression levels of the 2 MFN transgenes were comparable. Note low level of endogenous Mfn1 expression in the mouse brain and spinal cord. $n=3$ mice/genotype. (D) Flag immunostaining of MFN1 transgenic protein in 7-week-old mouse spinal cord showing punctate cytoplasmic staining consistent with mitochondria. DAPI stains nuclei. Scale bar: $100 \mu$ M. $n=3$ mice/genotype. (E) Body weight. Data are represented as mean \pm SEM. $n T g(n=8-32$ per time point), $M F N 2^{R 940}\left(n=24-63\right.$ per time point), and MFN2 ${ }^{R 940}$ :MFN1 ( $n=17-31$ per time point). Two-way ANOVA with Tukey's multiple comparisons test. ${ }^{*} P<$ 0.05 ; $^{* * *} P<0.0001$. MFN2 ${ }^{R 940}$ vs. MFN2R940:MFN1 (asterisks in black). nTg vs. MFN2 ${ }^{R 940}$ :MFN1 (asterisks in gray). (F) Survival curve. $\mathrm{nTg}(n=8-32$ per time point), MFN2 ${ }^{R 940}$ ( $n=24-63$ per time point), and MFN2 ${ }^{R 940}: M F N 1$ ( $n=17-31$ per time point). log-rank test with Bonferroni's correction. nTg vs. MFN2 ${ }^{R 940}, P<0.01 ;$ MFN2 ${ }^{R 940}$ vs. MFN2 ${ }^{R 940}: M F N 1, P<0.01$. nTg vs. MFN2 ${ }^{R 940}: M F N 1, P>0.05$ (not significant). (C) Open-field test (rearing). $\mathrm{nTg}(n=5), \operatorname{MFN2}^{R 940}(n=5)$, and MFN2 $2^{R 940}: \operatorname{MFN1}(n=10)$. (H) Rotarod testing. $\mathrm{nTg}(n=3-5)$, MFN2 $^{\text {R940 }}(n=3-5)$, and MFN2 ${ }^{R 940}:$ MFN1 $(n=3-10)$. $(\mathbf{I})$

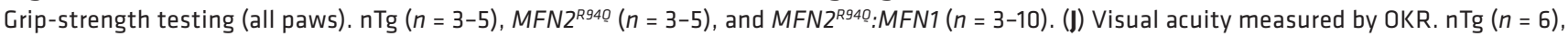
$\operatorname{MFN2}^{R 940}(n=7)$, and MFN2 ${ }^{R 940}:$ MFN1 $(n=4)$. In G-J, data are represented as mean \pm SEM. Two-way ANOVA with Tukey's test was used for multiple comparison. ${ }^{*} P<0.05 ;{ }^{* *} P<0.01 ;{ }^{* *} P<0.001 ;{ }^{* * *} P<0.0001$. 
rescuing fusion by promoting Mfn1:Mfn1-mediated fusion events. Interestingly, lentiviral expression of MFN2 ${ }^{\mathrm{R} 94 \mathrm{Q}}$ (but not MFN2 ${ }^{\mathrm{WT}}$ ) in SH-SY5Y neuroblastoma cells promoted mitochondrial clustering similar to that seen in the $M F N 2^{R 94 Q}$ mice, and clustering was exacerbated by the TAT-MP1 ${ }^{\text {Gly }}$ peptide (Figure $4, \mathrm{D}$ and E). This suggests that, because of the lower levels of endogenous MFN1 in this neuronal cell line, the further promotion of an HR1-HR2 tethering permissive conformation of $\mathrm{MFN} 2^{\mathrm{R} 94 \mathrm{Q}}$ enhanced the frequency of failed fusion events and mitochondrial clustering. In keeping with the idea that the MFN1/MFN2 ratio is the key determinant of sensitivity to the MFN2 ${ }^{\mathrm{R} 94 \mathrm{Q}}$ mutant, the mitochondrial aggregation effect of MFN2 ${ }^{\mathrm{R} 94 \mathrm{Q}}$ expression in $\mathrm{SH}-\mathrm{SY} 5 \mathrm{Y}$ cells was significantly rescued by increasing expression of MFN1 (Figure 4, D and E).

In summary, these data support the idea that $\mathrm{MFN} 2^{\mathrm{R} 94 \mathrm{Q}}$ is fusion incompetent and can suppress mitochondrial fusion in a dominant negative manner depending on the level of available fusion-competent MFN1 and MFN2 molecules in the cell. They further support the concept that the low levels of MFN1 in the nervous system potentially underlie the selective vulnerability of neurons in CMT2A (27).

Phenotypic rescue of $M F N 2^{R 94 Q}$ mice by augmenting $M F N 1$ expression in the nervous system. To examine whether altering the balance of MFN1 and MFN2 can mitigate the toxic effects of MFN2 ${ }^{\mathrm{R} 94 \mathrm{Q}}$, we generated mice expressing Flag-tagged human MFN1 under the mouse prion protein $(\mathrm{PrP})$ promoter to drive expression in the nervous system (Figure 5, A and B). Western blot confirmed the expression of Flag-tagged MFN1 in both brain and spinal cord at levels slightly lower than Thy1.2-driven MFN2 ${ }^{\mathrm{R} 94 \mathrm{Q}}$ (Figure $5 \mathrm{C}$ ). As noted before, endogenous Mfn1 protein was nearly undetectable in the nervous system of nontransgenic animals (Figure 5C). Despite the high level of Flag-MFN1 expression over endogenous Mfn1, normal punctate mitochondrial staining was observed in cell bodies (Figure 5D) and animals were phenotypically and histologically normal throughout their life spans.

We then crossed Prp-MFN1 mice with Thy1.2-MFN2 ${ }^{\text {R94Q }}$ mice to generate the double-transgenic (MFN2 $\left.{ }^{R 94 Q}: M F N 1\right)$ mice. Expression of MFN1 in the nervous system rescued the stunted growth and reduced survival seen in $M F N 2^{R 94 Q}$ mice (Figure 5, E and F). $M F N 2^{R 94 Q}$ : MFN1 mice appeared phenotypically normal with a normal gate (Supplemental Video 2) and did not exhibit clasping even at advanced ages (Supplemental Figure 7A). In comparison with $M F N 2^{R 94 Q}$ mice, $M F N 2^{R 94 Q}: M F N 1$ double-transgenic mice had marked improvement in rearing activity in open-field testing, rotarod fall latency, and grip strength (Figure 5, G-I). Visual acuity of $M F N 2^{R 94 Q}: M F N 1$ doubletransgenic mice was restored to the level of nontransgenic mice (Figure 5J). In summary, increasing the level of MFN1 in the nervous system was well tolerated and provided complete or near-complete phenotypic rescue of defects in locomotor activity, sensorimotor coordination, and vision loss in $M F N 2^{R 94 Q}$ mice.

Mitochondrial aggregation, axon degeneration, and transcriptomic signature rescued by MFN1 augmentation. We next determined whether increasing MFN1 levels in the nervous system could rescue the mitochondrial clustering, axon degeneration, and gliosis seen in Thy1.2-MFN2 ${ }^{R 94 Q}$ mice. To examine mitochondrial morphology and accumulations, we generated triple-transgenic mice carrying $M F N 2^{R 94 Q}, M F N 1$, and CFP-COX8A to fluorescently label mitochondria. Compared with $M F N 2^{R 94 Q}: C F P-C O X 8 A$ mice,
MFN2 ${ }^{\text {R94Q }}: M F N 1: C F P-C O X 8 A$ mice showed rescue of CFP-labeled mitochondrial clustering in hippocampus (Figure 6A). Immunofluorescence staining with anti-Flag and anti-p62 antibodies confirmed that MFN1 augmentation completely rescued the mitochondrial clustering caused by MFN2 ${ }^{\mathrm{R} 94 \mathrm{Q}}$ (Figure 6B and Supplemental Figure 7B). Fluoro-Jade staining demonstrated complete rescue of degenerating axons in the spinal cords of $M F N 2^{R 94 Q}: M F N 1$ mice in comparison with $M F N 2^{R 94 Q}$ mice (Figure 6C and Supplemental Figure 7C). The number of axonal neurofilament spheroids was significantly rescued in $M F N 2^{R 94 Q}: M F N 1$ mice when compared with $M F N 2^{R 94 Q}$ mice (Figure 6D and Supplemental Figure 7D). Finally, Gfap and Iba1 immunofluorescence staining were normalized in MFN2 ${ }^{R 94 Q}: M F N 1$ mice (Figure 6, E and F, and Supplemental Figure 7, $\mathrm{E}$ and $\mathrm{F}$ ), consistent with rescue of the microglial and astrocytic reactions seen in $M F N 2^{R 94 Q}$ mice.

To analyze transcriptome alterations in $M F N 2^{R 94 Q}$ mice, we performed RNA-Seq on the lumbar spinal cords of nontransgenic $M F N 2^{R 94 Q}$ and $M F N 2^{R 94 Q}: M F N 1$ animals. Principal component analysis (PCA) showed that the first principal component (PC1) separated the 3 genotypes, with nontransgenic and $M F N 2^{R 94 Q}$ : $M F N 1$ mice clustering more closely together and $M F N 2^{R 94 Q}$ clustering separately (Figure 6G). To define the pathways that drove this difference, we performed Gene Set Enrichment Analysis (GSEA) on the gene loadings ranked by the most positive to the most negative contributions to PC1 and found that oxidative phosphorylation and respiration electron transport were the pathways most significantly downregulated in PC1, indicating these drove the difference between the genotypes, and were rescued by MFN1 overexpression (Table 1). Overall, these data provide strong evidence that the ratio of MFN1 to MFN2 is a key determinant of the sensitivity of the nervous system to the dominant negative effects of mutant MFN2 expression and support the idea that elevating MFN1 levels to restore the MFN1/MFN2 balance is a viable therapeutic strategy for CMT2A (Figure 6H).

Finally, to further characterize whether MFN2 ${ }^{\mathrm{R} 94 \mathrm{Q}}$ functions as a dominant negative, we first examined whether MFN2 ${ }^{\mathrm{WT}}$ could also rescue the toxic effects of the mutant protein. As with MFN1 (Figure $4 \mathrm{D}$ ), increased expression of MFN2 ${ }^{\mathrm{WT}}$ using lentivirus decreased the amount of mitochondrial aggregates induced by mutant $\mathrm{MFN} 2^{\mathrm{R} 94} \mathrm{Q}_{\text {in }}$ SH-SY5Y cells in culture (Supplemental Figure 8A). To further determine whether increasing MFN2 ${ }^{\mathrm{WT}}$ could rescue the phenotype of $M F N 2^{R 94 Q}$ mice, we crossed the $M F N 2^{R 94 Q}$ to the $M F N 2^{W T}$ transgenic mice. We observed that MFN2 ${ }^{R 94 Q}: M F N 2^{W T}$ double-transgenic mice showed restored weight gain (Supplemental Figure 8B) and that their behavior appeared overtly normal and indistinguishable from that of MFN2 ${ }^{R 94 Q}:$ MFN1 mice. As in SH-SY5Y cells, hippocampal mitochondrial aggregation was rescued in the $M F N 2^{R 94 Q}: M F N 2^{W T}$ doubletransgenic mice (Supplemental Figure 8C). Finally, we observed that increasing MFN2 ${ }^{\mathrm{WT}}$ levels was able to rescue the axonal degeneration seen in the pyramidal tract of $M F N 2^{R 94 Q}$ mice (Supplemental Figure $8 \mathrm{D})$. These data strongly support that $\mathrm{MFN} 2^{\mathrm{R} 94 \mathrm{C}}$ mutant acts by a dominant negative mechanism that can be rescued by increasing the number of fusion-competent MFN1 or MFN2 molecules.

\section{Discussion}

In this study, we demonstrated that mice with transgenic expression of CMT2A-associated mutant MFN2 ${ }^{\mathrm{R} 94 \mathrm{Q}}$ (but not WT) developed 
A

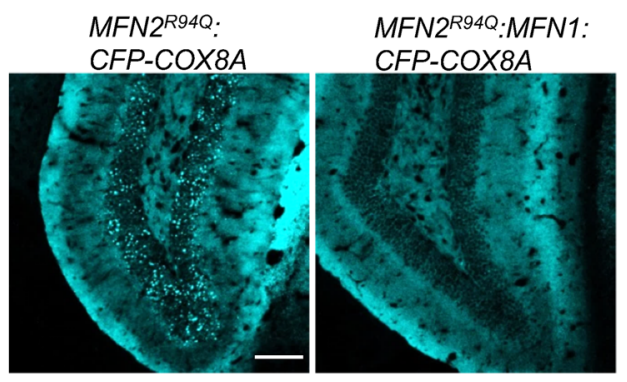

B

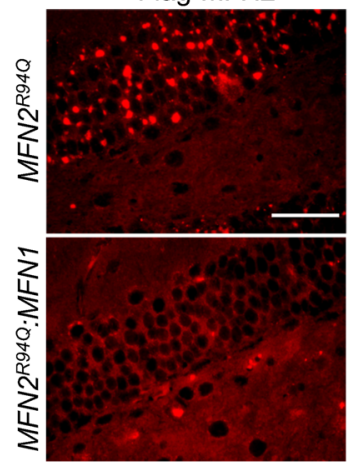

P62

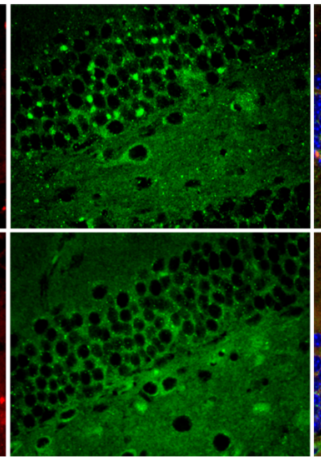

Overlay

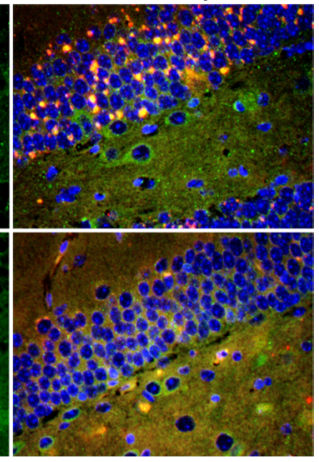

C

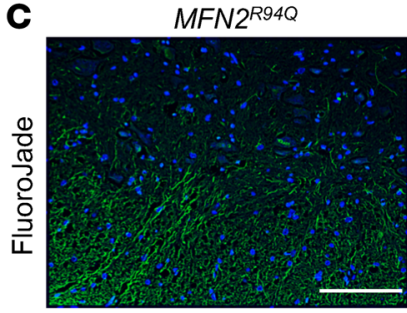

E

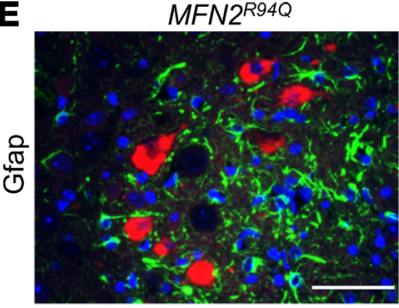

MFN2R94Q:MFN1
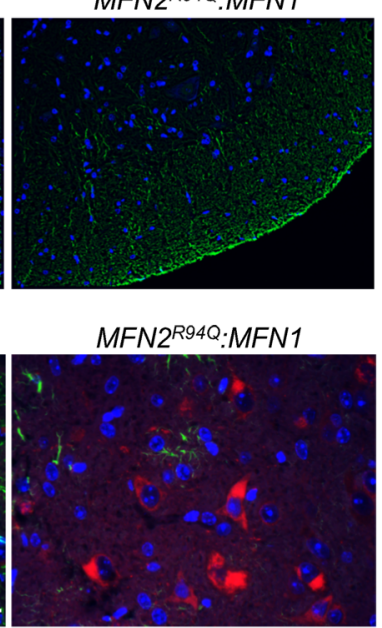

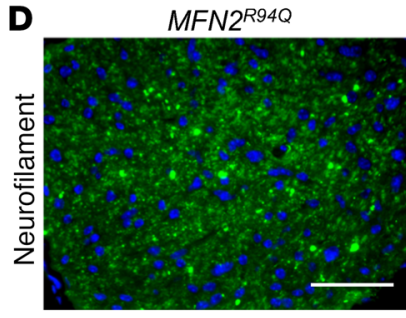

$\mathbf{F}$

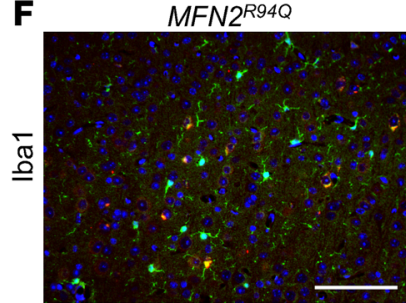

MFN2 ${ }^{R 94 Q}: M F N 1$

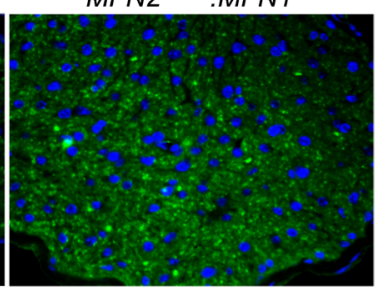

MFN2R94Q:MFN1

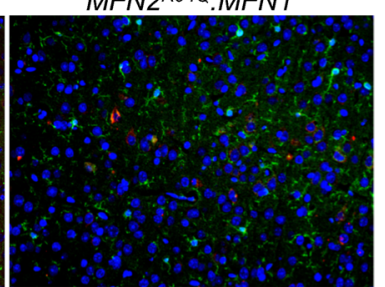

G

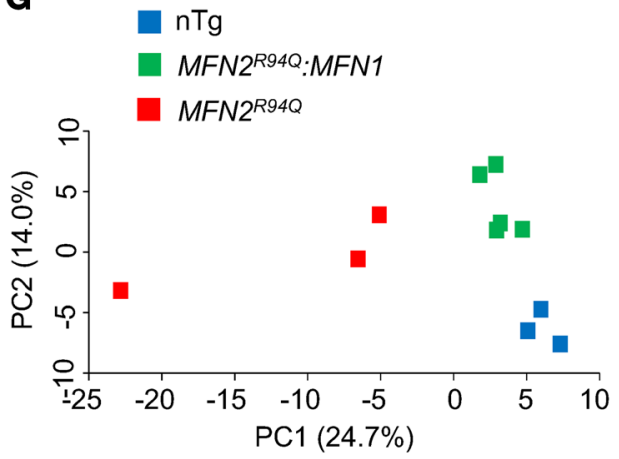

H Non-neuronal cells

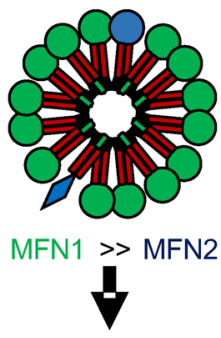

Normal function

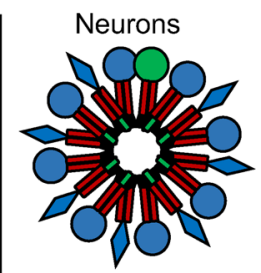

MFN1 $<$ MFN2

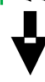

Decreased fusion Decreased transport Axon degeneration
Neurons + MFN1 augmentation

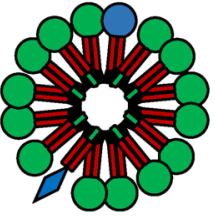

MFN1 > MFN2

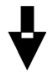

Restored function

Figure 6. Increased expression of MFN1 rescued the mitochondrial accumulation, axon degeneration, gliosis, and transcriptomic changes seen in MFN2 ${ }^{\text {Rg40 }}$ mice. (A) Mitochondrial accumulation labeled with CFP-COX8A in vivo (7-month-old mice). Scale bar: $100 \mu \mathrm{M} . n=2$ mice/genotype. The photograph for MFN$2^{R 940}:$ CFP-COX8A is the same as in Figure 3A. (B) Colocalization of clustered mitochondria with p62. Flag-MFN2R940 (red), p62 (green), DAPI (blue). Scale bar: 50 $\mu$ M. $n=3-4$ mice/genotype. (C) Degenerating axons stained with Fluoro-Jade in the spinal cord (5-month-old mice). Scale bar: $50 \mu M$. $n=2-3$ mice/genotype. (D) Neurofilament in cross sections of optic nerve stained with SMI32 antibody. Scale bar: $50 \mu \mathrm{M}$ (3-month-old mice). $n=3-4$ mice/genotype. (E) Astrogliosis in brains (brain stem shown here) of 5-month-old mice. Gfap (green), Flag (red), and DAPI (blue). Scale bar: $50 \mu$ M. $n=3$ mice/genotype. (F) Microglial staining in brains (cortex shown here) of 5-month-old mice. Iba1 (green), Flag (red), and DAPI (blue). Scale bar: $100 \mu \mathrm{M} . n=3$ mice/genotype. (C) PCA of RNA-Seq data of lumbar spinal cord (10 months old). $n=3-5$ mice/genotype. (H) Schematic diagram of a model for MFN1:MFN2 heterotypic interactions in cis in a torus-like conformation. The balance of MFN1 and MFN2 on mitochondria in different cell types determines the sensitivity to a dominant negative MFN2 mutant within a multimolecular complex (WT MFN shown with circular head, mutant MFN2 as a diamond). Altering the MFN1:MFN2 balance through increasing MFN1 protein in neurons restores mitochondrial fusion and other MFN functions despite the continued presence of mutant MFN2 ${ }^{\mathrm{Rg} 4 \mathrm{Q}}$.

a widespread axonopathy of the central and peripheral nervous systems, with defects in mitochondrial dynamics in neuronal cell bodies and axons. The phenotype resembled the severe early onset disease seen in most CMT2A patients and included some of the less common features, including optic atrophy with vision loss, long tract spinal cord involvement, and brain involvement $(15,16,18)$. These data support clinical observations that CMT2A is a broader disease of the nervous system, with long peripheral and central 
Table 1. Pathway analysis of MFN2 ${ }^{R 940}$ mice

$\begin{array}{lcc}\text { Pathways } & \begin{array}{c}\text { Changes } \\ \text { FDR } \\ \text { ( } q \text { value) }\end{array} \\ \text { REACTOME_RESPIRATORY_ELECTRON_TRANSPORT } & \text { Down } & 0.0092 \\ \text { KEC__OXIDATIVE_PHOSPHORYLATION } & \text { Down } & 0.0083 \\ \text { GO__RANSFERASE_ACTIVITY_TRANSFERRING_SULFUR_CONTAINING_GROUPS } & \text { Down } & 0.0104 \\ \text { KECG_PARKINSONS_DISEASE } & \text { Down } & 0.0213 \\ \text { REACTOME_INTERFERON_ALPHA_BETA_SIGNALING } & \text { Down } & 0.0227 \\ \text { REACTOME_CHOLESTEROL_BIOSYNTHESIS } & \text { Down } & 0.0392 \\ \text { GO_RECULATION_OF_CELL_DIFFERENTIATION } & \text { Up } & 0.0364 \\ \text { GO_RECULATION_OF_CROWTH } & \text { Up } & 0.0358\end{array}$

CSEA of pathways using ranked gene loadings contributing to PC1 from PCA. GO, Gene Ontology. ${ }^{A} F D R \leq 0.05$.

axons most sensitive to the effects of mutant MFN2 (18, 34, 49-51). Several previous mouse models of CMT2A from mutant MFN2 have been generated, but none with the diffuse axonopathy seen in Thy1.2-MFN2 ${ }^{R 94 Q}$ mice, particularly in the central nervous system. HB9-Mfn $2^{T 105 M}$ transgenic mice developed hind limb defects, but this was distinct from human phenotypes in that it was only seen in homozygotes and was congenital and nonprogressive, likely because of promoter inactivation after development (28). Mice expressing MFN2 ${ }^{\mathrm{R} 94 \mathrm{Q}}$ under the neuron-specific enolase (NSE) promoter showed motor deficits starting at 5 months of age (29, 52 ), and mice with MFN2 ${ }^{\mathrm{T} 105 \mathrm{M}}$ expressed inducibly in the nervous system under the Rosa locus developed only mild gait defects (31). Finally, MFN2 mutant knockin mice either had no reported phenotype (27) or mild late-onset deficits (30). These differences are likely due to the use of different promoters and the requirement for at least mild overexpression to promote a phenotype. It is notable that, while we observed axonal degeneration in the brain and spinal cord of Thy1.2-MFN2 $2^{R 94 Q}$ mice, we observed axonal atrophy in the distal peripheral nerve without axon loss at a time when the animals manifested a profound behavioral phenotype. Axonal atrophy without degeneration has been observed in a prior CMT2A model (29) as well as other mouse models of axonal CMT $(37,38)$. These data suggest that a critical threshold of mutant MFN2 expression is required to produce peripheral axonal degeneration in mice, which may be due to species-specific factors, such as shorter axon length or uncharacterized genetic modifiers. Likewise, they suggest that the profound behavioral phenotype in Thy1.2-MFN $2^{R 94 Q}$ mice is presumably driven by a combination of vision loss and sensory-motor axonal damage in brain and spinal cord. While transgene insertion into the Y chromosome was a drawback of the R94Q-44 line, as female mice could not be studied, clinical data from humans do not support a sex difference in phenotypes, and while the R94Q-62 line was too severe to maintain, it showed similar pathology in both males and females.

The most striking pathologic feature in Thy1.2-MFN2 ${ }^{R 94 Q}$ mice was mitochondrial clustering and aggregation in neuronal cell bodies and proximal and distal axons. Similar abnormal mitochondrial size, morphology, and clustering has been reported in the nerve biopsies from CMT2A patients $(40,41)$. Mutant-specific mitochondrial aggregation was also observed in brain and spinal cord of $M F N 2^{T 105 M}$ transgenic mice (31) and neurons cultured from $M F N 2^{T 105 M}$ transgenic mice (28). It has also been a consistent feature in tissue culture models $(26,27,32)$. Mitochondrial accumulations in cell bodies were labeled with p62, indicating they were marked for degradation via mitophagy (53); however, we did not see a difference in Parkin localization or recruitment of mitochondria to lysosomes in MEFs expressing MFN2 ${ }^{\mathrm{R} 94 \mathrm{Q}}$, suggesting that mitochondrial accumulations did not result from a mutantinduced block in mitophagy.

What are the causes and consequences of mitochondrial aggregation? Our data suggest that effects of mutant $\mathrm{MFN} 2^{\mathrm{R} 94 \mathrm{Q}}$ on mitochondrial fusion $(27,32)$ and engagement with the transport apparatus $(3,26,32)$ are likely to drive mitochondrial aggregation (see Figure 4A). While there are several models of MFN2 structure and topology, all involve an initial tethering step, followed by GTP hydrolysis as a key step mediating membrane fusion $(43,45-48)$. The most well-studied of these models involves tethering via a C-terminal HR2 domain on adjacent mitochondria $(43,45,54)$. In this model, MFN exists either in a fusion-nonpermissive conformation through HR1-HR2 intramolecular binding or in fusion-permissive conformation through intermolecular HR2 binding. By this model, GTPase domain mutants (such as MFN2 ${ }^{\mathrm{R} 94 \mathrm{Q}}$ ) are predicted to be competent for initial HR2-dependent tethering of adjacent mitochondria, but unable to mediate fusion because of GTPase dysfunction. Aggregates of unfused mitochondria would then result from tethered mitochondria that were unable to complete fusion or engage transport apparatus. This is supported by the observation that a peptide that allosterically modulates HR1-HR2 interactions led to increased MFN2-mediated mitochondrial clustering in a neuronal cell line (Figure 4D). However, it is important to note that alternative models exist whereby MFNs have a different membrane topology $(47,55)$, and other models where initial tethering takes place via the GTPase domain rather than the C-terminal HR2 domain $(46,48)$, so further structural biology studies are needed to reconcile these different models. Regardless of the precise molecular mechanism, mitochondrial aggregation accompanied by decreased mitochondrial fusion and axonal transport/localization likely drives axonal degeneration in Thy1.2-MFN2 ${ }^{R 94 Q}$-expressing mice, as numerous prior in vitro studies support that degeneration results from local axonal energy failure due to abnormal mitochondrial function, interaction with other organelles, including ER and lysosomes, and improper localization to sites of energy demand $(2,6,26,27,52,56)$. Of note, a recent study showed that the $\mathrm{MFN} 2^{\mathrm{R} 94 \mathrm{Q}}$ mutant in flies led to similar cytoplasmic aggregates of unfused mitochondria and axonal pathology, supporting the dominant negative tethering model for the action of this and other GTPase domain mutants (57). Interestingly, the authors found that 2 other mutants (L76P and R364W) showed evidence of enhanced fusion, but surprisingly, still caused mitochondrial dysfunction and altered axonal mitochondrial localization. This was consistent with earlier findings that the L76P mutant disrupted mitochondrial transport $(32,58)$, but not fusion (27). Further work will need to be done to determine whether MFN1 augmentation will mitigate the toxic effects of other dominant MFN2 mutants that may have different mechanisms of action.

In addition to modeling the toxic effects of mutant MFN2 that lead to CMT2A, we provided what we believe is the first in vivo proof of concept that restoring MFN1:MFN2 balance by augmenting 
levels of MFN1 in the nervous system is a viable therapeutic approach for CMT2A, as Thy1.2-MFN2 ${ }^{\text {R94Q }}$ Prp-MFN1 doubletransgenic animals showed near complete rescue of ocular, neuromuscular, and histologic phenotypes seen in Thy1.2-MFN2 ${ }^{R 94 Q}$ mice. While $M f n 1$ - or $M f n 2-n u l l$ mice are embryonic lethal due to placental defects (1), conditional removal of Mfn2, but not Mfn1, postnatally led specifically to neurodegeneration $(10,23,24)$, likely due to the fact that Mfn1 levels are very low in the nervous system (27). MFN1 and MFN2 are 80\% identical (22) and are capable of heterodimer formation (1), and MFN1 expression can rescue mitochondrial fusion and transport defects caused by the loss of MFN2 in MEFs and DRG neurons $(26,27)$, but not other functions, such as altered ER-mitochondrial interactions (2). Therefore, these data support the concepts that (a) the origin of nervous system-specific defects in CMT2A is due to the relative lack of MFN1 expression and (b) functions that are MFN2 specific are not critical for the toxicity of MFN2 mutants in CMT2A, as they would presumably not be rescued by restoring MFN1:MFN2 balance.

We also observed that increasing MFN2 levels in neurons (in SH-SY5Y cells or by generating double-transgenic $M F N 2^{R 94 Q}: M F N 2^{W T}$ mice) could similarly rescue the dominant negative effect of mutant $\mathrm{MFN} 2^{\mathrm{R} 94 \mathrm{Q}}$. This further supports the idea that the relative number of functional MFN molecules (either MFN1 or MFN2) in a tissue is the key driver of toxicity of mutant $\mathrm{MFN} 2^{\mathrm{R} 94 \mathrm{Q}}$ and that increasing either MFN1 or MFN2 could be used therapeutically in CMT2A. However, for approaches increasing endogenous gene expression, augmenting MFN1 would be preferable, as it would effectively change the ratio of functional MFN molecules, while augmenting MFN2 expression would have no effect on this ratio or could worsen the phenotype.

It is unknown why MFN1 levels are very low in the adult nervous system, but the fact that Prp-MFN1 transgenic mice are phenotypically normal indicates that MFN1 overexpression at levels equivalent to MFN2 is well tolerated. Likewise, increased levels of MFN2 in the Thy1.2-MFN2 ${ }^{W T}$ micewerealsowelltoleratedand couldsimilarlyrescue the toxic effects of mutant MFN2 expression. This raises the possibility that augmenting MFN1 or MFN2 expression in the nervous system may have broader therapeutic applications beyond CMT2A. Impaired mitochondrial dynamics, including fusion, fission, and transport, have been implicated in other forms of CMT (59-62) and many other neurodegenerative diseases, including Parkinson's disease, Huntington's disease, amyotrophic lateral sclerosis, and Alzheimer's disease $(7,8,63)$. Indeed, a recent report indicated that increasing MFN2 levels in neurons was protective in a model of amyotrophic lateral sclerosis, suggesting that $M F N 2^{W T}$ mice may be intrinsically more resistant to neurodegeneration (64). Although upstream mechanisms vary across these neurodegenerative diseases, augmenting MFN1 or MFN2 could mitigate mitochondrial dysfunction that occurs as a shared pathophysiology in neurodegeneration.

\section{Methods}

\section{Generation of transgenic mice}

Thy1.2-Flag-MFN2 ${ }^{W T}$ or Thy1.2-Flag-MFN2 ${ }^{R 94 Q}$ mice. An XhoI site was inserted using QuikChange (Agilent) to remove MFN2 3' UTR in the pEGFP-HA-MFN2 ${ }^{\text {WT }}$ and pEGFP-HA-MFN2 ${ }^{\text {R94Q }}$ cDNA constructs (32). The HA tag was then replaced with the Flag tag using In-Fusion cloning (Clontech). The resulting Flag-MFN2 ${ }^{\text {WT }}$ or Flag-MFN2 ${ }^{\mathrm{R} 94 \mathrm{Q}}$ was cut out from the pEGFP construct and subcloned into the Thy1.2 promoter construct (Addgene, cata$\log 20736)$ to make a Thy1.2-Flag-MFN2 ${ }^{\mathrm{WT}}$ or Thy1.2-Flag-MF$\mathrm{N} 2^{\mathrm{R} 94 \mathrm{Q}}$ construct using the XhoI restriction sites (65). The plasmid was linearized with EcoRI and PvuI digestion, gel purified, and filter sterilized for pronuclear injection. The $M F N 2^{W T}$ or MFN2 ${ }^{\text {R94 }}$ transgenic mice were created on the C57BL/6J background (The Jackson Laboratory) with standard pronuclear injection procedure. Genotyping of founder mice was done using PCR. Primers were as follows: forward, 5'-GAAGTTCGCCTTGATGCCGTTCTTCTG; reverse, 5'-TCCAGTTCTGCATTCCTGTACG. Thy1.2-Flag-MFN2 ${ }^{R 94 Q}$ line 44 heterozygous mice, Thy1.2-Flag-MF$N 2^{W T}$ mice, and PrP-Flag-MFN1 mice were generated in house and deposited in The Jackson Laboratory (JAX stock 29745, JAX stock 032728, and JAX stock 033391, respectively).

PrP-Flag-MFN1 mice. Human MFN1 cDNA cloned into pBluescriptR plasmid was obtained from Open Biosystems (MHS10109203753). An AgeI-Kosak-ATG-FLAG sequence was inserted after the 5' UTR, and an XhoI site was inserted before the 3' UTR of human MFN1 cDNA using QuikChange. Flag-MFN1 was then cut out from the pBluescriptR plasmid with AgeI and XhoI restriction sites and subcloned into the PrP promoter construct. The final PrP-FlagMFN1 plasmid was linearized with NotI restriction enzyme digestion. The linearized plasmid was used for pronuclear injection to create transgenic mice on the C57BL/6J background. Genotyping of founder mice was done using PCR. Primers were as follows: forward, 5'-CAATTCTAGTTTTCTCTTTTTGTTGTTTTAAAGGACTCC; reverse, 5'- GGGAGAACTTTATCCCACAACA.

MFN2 ${ }^{R 94 Q}:$ MFN1 and MFN2 ${ }^{\text {R94Q }}$ : MNF2 ${ }^{\text {WT }}$ double-transgenic mice. $M F N 2^{R 94 Q}$ mice were crossed with either MFN1 mice or MFN2 ${ }^{W T}$ mice to generate $M F N 2^{\text {R94Q }}$ : MFN1 or MFN2 ${ }^{\text {R94Q }}$ MFN2 ${ }^{\text {WT }}$ heterozygous double-transgenic mice. To fluorescently label mitochondria in vivo, $M F N 2^{R 94 Q}$ mice or MFN2 ${ }^{R 94 Q}$ : MFN1 mice were crossed with Thy1-CFP/ COX8A mice from The Jackson Laboratory to make $M F N 2^{R 94 Q}$ : CFP/ COX8A mice or MFN2 ${ }^{R 94 Q}$ : MFN1: CFP/COX8A mice.

\section{Mouse behavioral analysis}

Rotarod test. The rotarod was used to measure motor function and coordination. Mice were placed on a rod elevated 18 inches above a soft surface. For each rotarod session, the rod was set at a constant speed of $5 \mathrm{rpm}$ with a cut-off time at 4 minutes. Each session consisted of 3 trials with a 30-minute intertrial period in which mice were placed back in their home cages. The latency for the mice to fall off the rod was averaged across the 3 trials.

Grip-strength test. This test measures grip-force ability of the forelimbs and hind limbs. For measuring tensile force, the mouse was held by the base of the tail and allowed to grip a metal grid either with its forepaws alone or with both forepaws and hind paws. The mouse was then gently pulled horizontally to exert the tensile force. The peak force (in $g$ ) at the point the mouse released its grip was recorded through a digital force gauge attached to the metal grid. Three successive measurements were averaged for both the forelimbs and the 4-paws grip strengths.

Open-field test. Spontaneous motor activity of the mice was measured in the open-field test. Mice were individually placed into a clear Plexiglas chamber (16 inches long $\times 16$ inches wide $\times 15$ inches high) surrounded by 2 bands of photobeams and optical sensors that mea- 
sured horizontal locomotor and vertical rearing activity. Animals moved freely within the enclosure for 60 minutes. Movement was detected as breaks within the photobeam matrices and automatically recorded using PAS software (San Diego Instruments).

Visual acuity test. Visual acuity measured by OKR was conducted on both mutated and control mice. The test was done in an observerblinded fashion as reported previously (66).

Western blot analysis. Mouse brain or spinal cord tissue was lysed with RIPA buffer and quantified using BCA protein assay (Pierce). Protein samples were separated on $10 \%$ precast polyacrylamide gels and transferred to nitrocellulose blots. Primary antibodies for Western blot included anti-Flag (MilliporeSigma, catalog F3165), anti-MFN2 (Abcam, catalog ab56889), anti-MFN1 (Proteintech, catalog 13798-1AP), and anti-GAPDH (MilliporeSigma, catalog G8795). Signals were detected using fluorescent secondary antibodies and the Odyssey imager from LI-COR.

Immunohistochemistry analysis of brain, spinal cord, and optic nerve. Tissue were fixed in $4 \%$ paraformaldehyde (PFA) ( $\mathrm{pH} 7.4$ ) for 5 days for brain and spinal cord and for 2 days for optic nerve. After fixation, tissues were embedded in paraffin and $4 \mu \mathrm{m}$ thick serial sections were cut, including coronal sections for brain, cross sections for spinal cord, and both cross and longitudinal sections for optic nerve. Sets of sections were deparaffinized using xylene and then hydrated with decreasing concentrations of ethanol. After deparaffinization, antigen retrieval was performed by submerging the sections in citrate buffer $(\mathrm{pH}$ 6.0) and microwaving at $800 \mathrm{~W}$ for 10 minutes. For SMI32 staining, the microwave step was followed by 5 minutes incubation in $90 \%$ formic acid. For immunostaining, the sections were treated with $1 \% \mathrm{H}_{2} \mathrm{O}_{2} /$ methanol and blocked with $10 \%$ horse serum, followed by overnight incubation with primary antibodies. The next day, sections were incubated with fluorescent secondary antibodies for 4 hours at room temperature. Nuclei were stained with DAPI and mounted with ProLong Gold Antifade Mountant (Thermo Fisher Scientific). Positive and negative controls were run along each batch of sections processed.

Primary antibodies were as follows: anti-FLAG (1:500, catalog F3165, Sigma-Aldrich), anti-COXIV (1:200, catalog ab16056, Abcam Inc.), anti-p62 (1:500, catalog BML-PW9860-0100, Enzo Life Sciences), anti-ubiquitin (1:500, catalog MAB1510, MilliporeSigma), anti-neurofilament $\mathrm{H}$ nonphosphorylated (SMI 32, 1:500, catalog 801701, Biolegend), anti-NeuN (1:200, catalog MAB377, MilliporeSigma), anti-GFAP (1:500, catalog Z0334, Dako-Agilent Technologies Inc.), anti-Iba1 (1:250, catalog 019-19741, Wako). Secondary antibodies were as follows: 1:1000, Alexa Fluor 488 (Molecular Probes, cata$\log$ A11034, catalog A11029) and Alexa Fluor 594 (Molecular Probes, catalog A11037, catalog A11032).

Stained sections were evaluated and imaged with an Olympus BX51 Upright Microscope. The quantification of fluorescent signals was performed using Image J software (NIH) with the threshold and the analyze particles functions. For optic nerve, the number of neurofilament spheroids (size more than 50 pixel $^{2}$ ) was quantified. For neuron counts, the numbers of NeuN-positive cells were counted in brain and spinal cord. For astrogliosis analysis, GFAP-positive signals were quantified as percentage of area. For microgliosis analysis, numbers of Iba1-positive cells were counted. To analyze mitochondrial clusters, numbers of p62-positive mitochondrial clusters per region of interest (ROI) $\left(0.01 \mathrm{~mm}^{2}\right)$ in the hippocampus were quantified.
Immunohistochemistry analysis of retinal whole mount and retinal sections. Retinal whole mount was prepared according to published protocols (67). Briefly, eyeballs were fixed in 4\% PFA for 1 hour and transferred to PBS ( $\mathrm{pH}$ 7.35-7.45) for dissecting. Under the dissecting microscope, the cornea and lens were removed and 4 equal cuts were made to flatten the eyeball. Neural retina was dissected out, transferred to filter paper, and fixed for 1 hour in the same fixative. The neural retina was washed with $\mathrm{PBS}$, infiltrated with gradient sucrose $(10 \%, 20 \%$, and $30 \%)$, and left in $30 \%$ sucrose overnight at $4^{\circ} \mathrm{C}$. On the next day, the retina was frozen and thawed 3 times in an Eppendorf tube with $30 \%$ sucrose on dry ice. The retina was washed with PBS and processed for immunofluorescence staining. Retinal sections were prepared according to published protocols (67). Briefly, eyes were removed and immersed in 2\% PFA for 1 hour. Eyes were then infiltrated with sucrose and embedded in OCT. Horizontal frozen sections $(12 \mu \mathrm{m})$ were cut on a cryostat. The primary antibodies used were anti-neurofilament (RT97, 1:1,000, catalog CBL212, MilliporeSigma) and anti-Brn3a (1:200, catalog sc-31984, Santa Cruz Biotechnology Inc.) for RGCs.

Retinas were examined, and images were taken using the Leica DM6000B microscope (Leica Microsystems). The numbers of RGC cells (Brn3a positive) in the retinal whole mount were quantified. Four images in the center and in the periphery were taken, respectively, per animal. Four or five animals were used per genotype. The quantification was performed using Image J software with the threshold and the analyze particles functions.

NMJ staining. Quadriceps muscle was dissected from mice and post-fixed with $4 \%$ PFA on ice for 1 hour. After PBS wash, muscle was embedded with $4 \%$ low melting agarose. Serial sections ( $70 \mu \mathrm{m}$ thick) were cut. Agarose sections were placed in a 24-well plate, blocked with $5 \%$ normal donkey serum (diluted in PBS) for 30 minutes, and stained with $0.5 \mu \mathrm{g} / \mathrm{ml} \alpha$-bungarotoxin (catalog B-13423, Thermo Fisher Scientific) for 1 hour. The stained sections were mounted on slides with ProLong Gold Antifade Mountant. Z-stack images were taken using a Nikon Eclipse Ti microscope.

Fluoro-Jade staining of degenerating axons. Sets of paraffin sections $(4 \mu \mathrm{m})$ were deparaffinized using xylene and then hydrated with decreasing concentrations of ethanol. The slides were immersed in the following solutions in sequence: PBS, 5 minutes; $1 \% \mathrm{NaOH}$ in $80 \%$ ethanol, 5 minutes; 70\% ethanol, 2 minutes; $\mathrm{H}_{2} \mathrm{O}, 2$ minutes; $0.06 \%$ $\mathrm{KMnO} 4$ in $\mathrm{H}_{2} \mathrm{O}, 17$ minutes; $\mathrm{H}_{2} \mathrm{O}, 2$ minutes; 0.001\% Fluoro-Jade C (catalog AG235, MilliporeSigma) in $0.1 \%$ acetic acid with DAPI, 30 minutes; and $\mathrm{H}_{2} \mathrm{O}, 3$ times, 2 minutes per time. Then slides were dried by draining excess water and placed on a slide warmer at $50^{\circ} \mathrm{C}$ for $10 \mathrm{~min}$ utes. Finally, slides were coverslipped using DPX mounting medium. Images were taken using an Olympus BX51 Upright Microscope. Fluoro-Jade signals were quantified with ImageJ software. After background subtraction, Fluoro-Jade-positive signals per ROI were quantified and presented as pixle ${ }^{2} / \mathrm{mm}^{2}$ ROI.

Toluidine blue staining of spinal cord and tibial nerve plastic sections. A $1 \mathrm{ml}$ long segment of spinal cord (between thoracic and lumbar region) or distal tibial nerve were harvested for processing of epoxy resin blocks. The specimens were fixed in $3 \%$ glutaraldehyde in $0.1 \mathrm{M}$ phosphate buffer, osmicated in $2 \%$ osmium tetroxide in $0.1 \mathrm{M}$ phosphate buffer, dehydrated in increasing graded solutions of ethanol and acetone, and finally embedded in epoxy resin. Sections $(0.5 \mu \mathrm{m})$ were cut and stained with toluidine blue. Axon counts and axon area 
measurements were quantified as previously described in detail (68). Briefly, with ImageJ software, the threshold function was used to highlight axoplasm only on whole nerve sections; the analyze particle function was then used to count the number of myelinated axons and their cross-sectional areas in each nerve.

Imaging analysis of mitochondria and mitophagy in cultured cells. Mitochondrial labeling and mitophagy assay were performed as previously described (43). Briefly, MEFs were grown on coverslips. WT MEFs were infected with lentivirus expressing HA-MFN2 ${ }^{\mathrm{WT}}$ or HA-MFN2 ${ }^{\mathrm{R} 94 \mathrm{Q}}$ (32). Mitochondria in MEFs were labeled with MiotoTracker Green (200 nM, Invitrogen, catalog M7514) and tetramethylrhodamine, ethyl ester (TMRE; 200nM, Invitrogen, catalog T-669). For mitophagy studies, MEFs were stained with MitoTracker Green and transfected with an mCherry-Parkin-expressing adenovirus vector or stained with LysoTracker Deep Red (50 nM, Invitrogen, cata$\log$ L-7528); carbonyl cyanide-4-phenylhydrazone (FCCP) $(10 \mu \mathrm{M}$, 1 hour) was applied as a positive control. Images were taken using a Nikon Ti confocal microscope with a ×60 1.3 NA oil-immersion objective. Imaging analysis was performed as previously described (43).

For in vitro rescue assay, Mfn1/Mfn2-null MEFs (ATCC, CRL-2994) or Mfn2-null MEFs (ATCC, CRL-2993) were treated with allosteric MFN2 modulating peptide (TAT-MP1 ${ }^{\text {Gly }}$ ), and mitochondria were labeled with MitoTracker Green. SH-SY5Y cells (ATCC, CRL-2266 ${ }^{\mathrm{TM}}$ ) were infected with lentivirus (HA-MFN2 R94Q in CCImCh) expressing HA-MFN2 ${ }^{\mathrm{R} 94 \mathrm{Q}}$ with IRES-mitoCherry alone or in combination with TAT-MP1 ${ }^{\text {Gly }}$ treatment or lentivirus expressing Mfn1. After 96 hours, cells were fixed with 4\% PFA and kept in PBS. Images were taken using a Nikon Eclipse Ti microscope. Image analysis was performed using Image software to count the number of cell aggregates. Peptide TAT-MP1 ${ }^{\text {Gly }}$ was obtained in house and synthesized as previously reported (40).

RNA-Seq and pathway analysis. RNA was purified from spinal cord lumbar segment using TRIzol and the Purelink RNA Mini Kit (Thermo Fisher Scientific, 12183018A). RNA-Seq library was prepared using TruSeq Stranded mRNA Library Preparation Kit (Illumine, RS-1222102). Single-end 75 bp sequencing was performed with an Illumina NextSeq 500 at the Cedars-Sinai Genomics Core Facility. The FASTQ files were uploaded to Illumina BaseSpace for alignment to the mouse genome build mm10 using TOPHAT. The resulting BAM files were imported to Partek software to generate RPKM values and for differential expression analysis. The RPKM values were uploaded to Gene Cluster 3.0 for PCA. The genes that fell into PC1 were uploaded to GSEA for pathway analysis (69).

Accession code. All original raw and processed RNA-Seq data were deposited in the NCBI's Gene Expression Omnibus database (GEO GSE116863).

Statistics. All statistical analysis was performed using GraphPad Prism software (version 6.0). Statistical methods are indicated in the figure legends. Data are presented as mean \pm SEM $(n \geq 3)$. Differences are considered statistically significant at $P<0.05$. Unpaired 2-tailed Student's $t$ test was used for 2-group comparisons, unless it is stated that a 1-tailed $t$ test was used. Either 1- or 2-way ANOVA with Tukey's test was used for multiple comparisons. The log-rank test with Bonferroni's correction was used for survival curve comparison. The KolmogorovSmirnov test was used to compare frequency distribution.

Study approval. All animal experiments were performed after prior approval by the IACUC at Cedars-Sinai Medical Center and conformed to the policies and procedures of the Comparative Medicine Department, Cedars-Sinai Medical Center, as well as the NIH Guide for the Care and Use of Laboratory Animals (National Academies Press, 2011).

\section{Author contributions}

YZ and RHB planned, designed, and interpreted the experiments. $\mathrm{YZ}$ coordinated the project and was involved in most experiments as well as data collection and statistical analysis. SC and SB generated and characterized the transgenic mice. SC, JL, and MV established and maintained mouse colonies. $\mathrm{YZ}$ and SC analyzed the mouse behavioral data. YZ, SC, AKMGM, and JL performed tissue collection and analysis. SB embedded and processed the tissues. AKMGM performed tissue immunohistochemistry. YZ did all of the imaging and quantification. AF and GWD performed MEF mitochondrial labeling and mitophagy assay and analysis. YZ performed the SH-SY5Y cell assay, imaging, and quantification. $\mathrm{BL}$ and SW performed OKR tests and collected, processed, and imaged the eye tissues. CML performed plastic sections processing, imaging, and quantification. $\mathrm{YZ}, \mathrm{SB}, \mathrm{RH}$, and $\mathrm{RHB}$ performed RNA-Seq and analysis. YZ and RHB wrote the manuscript.

\section{Acknowledgments}

The authors thank Makoto Katsumata and the Cedars-Sinai Rodent Genetics Core for generating transgenic mice, J.-P. Vit of the Cedars-Sinai Behavior Core for performing the mouse behavioral tests, and Jacqueline O'Rourke, Deepti Lall, Arwin AghamalekySarvestany, and Michael Guerrero for technical assistance. This study was supported by NIH grants NSO55980 and NS097545, the Muscular Dystrophy Association (no. 352536), and the Charcot-MarieTooth Association (to RHB); NIH grant AG056678 (to RH); NIH grant R35HL135736 (to GWD); and a McDonnell Center for Cellular and Molecular Neurobiology postdoctoral fellowship (to AF).

Address correspondence to: Robert H. Baloh, Center for Neural Science and Medicine, Board of Governors Regenerative Medicine Institute, Department of Neurology, Cedars-Sinai Medical Center, 8700 Beverly Boulevard, Los Angeles, California 90048, USA. Phone: 310.423.5152; Email: robert.baloh@csmc.edu.
1. Chen H, Detmer SA, Ewald AJ, Griffin EE, Fraser SE, Chan DC. Mitofusins Mfn1 and Mfn2 coordinately regulate mitochondrial fusion and are essential for embryonic development. J Cell Biol. 2003;160(2):189-200.

2. de Brito OM, Scorrano L. Mitofusin 2 tethers endoplasmic reticulum to mitochondria. Nature. 2008;456(7222):605-610.

3. Misko A, Jiang S, Wegorzewska I, Milbrandt J, Baloh RH. Mitofusin 2 is necessary for trans- port of axonal mitochondria and interacts with the Miro/Milton complex. JNeurosci. 2010;30(12):4232-4240.

4. Chen $\mathrm{H}$, et al. Mitochondrial fusion is required for mtDNA stability in skeletal muscle and tolerance of mtDNA mutations. Cell. 2010;141(2):280-289.

5. Chen Y, Dorn GW. PINK1-phosphorylated mitofusin 2 is a Parkin receptor for culling damaged mitochondria. Science. 2013;340(6131):471-475.

6. Baloh RH. Mitochondrial dynamics and peripher- al neuropathy. Neuroscientist. 2008;14(1):12-18.

7. Misgeld T, Schwarz TL. Mitostasis in neurons: maintaining mitochondria in an extended cellular architecture. Neuron. 2017;96(3):651-666.

8. Burté F, Carelli V, Chinnery PF, Yu-Wai-Man P. Disturbed mitochondrial dynamics and neurodegenerative disorders. Nat Rev Neurol. 2015;11(1):11-24.

9. Tanaka A, et al. Proteasome and p97 mediate mitophagy and degradation of 
mitofusins induced by Parkin. J Cell Biol. 2010;191(7):1367-1380.

10. Lee $S$, et al. Mitofusin 2 is necessary for striatal axonal projections of midbrain dopamine neurons. Hum Mol Genet. 2012;21(22):4827-4835.

11. Wang X, et al. Impaired balance of mitochondrial fission and fusion in Alzheimer's disease. J Neurosci. 2009;29(28):9090-9103.

12. Manczak M, Calkins MJ, Reddy PH. Impaired mitochondrial dynamics and abnormal interaction of amyloid beta with mitochondrial protein Drp1 in neurons from patients with Alzheimer's disease: implications for neuronal damage. Hum Mol Genet. 2011;20(13):2495-2509.

13. Züchner S, et al. Mutations in the mitochondrial GTPase mitofusin 2 cause Charcot-MarieTooth neuropathy type 2A. Nat Genet. 2004;36(5):449-451.

14. Nicholson GA, et al. Severe early-onset axonal neuropathy with homozygous and compound heterozygous MFN2 mutations. Neurology. 2008;70(19):1678-1681.

15. Shy ME, Patzkó A. Axonal Charcot-Marie-Tooth disease. Curr Opin Neurol. 2011;24(5):475-483.

16. Züchner S, et al. Axonal neuropathy with optic atrophy is caused by mutations in mitofusin 2 . Ann Neurol. 2006;59(2):276-281.

17. Zhu D, Kennerson ML, Walizada G, Züchner S, Vance JM, Nicholson GA. Charcot-Marie-Tooth with pyramidal signs is genetically heterogeneous: families with and without MFN2 mutations. Neurology. 2005;65(3):496-497.

18. Chung KW, et al. Early onset severe and late-onset mild Charcot-Marie-Tooth disease with mitofusin 2 (MFN2) mutations. Brain. 2006;129(Pt 8):2103-2118.

19. Del Bo R, et al. Mutated mitofusin 2 presents with intrafamilial variability and brain mitochondrial dysfunction. Neurology. 2008;71(24):1959-1966.

20. Rouzier C, et al. The MFN2 gene is responsible for mitochondrial DNA instability and optic atrophy 'plus' phenotype. Brain. 2012;135(Pt 1):23-34.

21. Sawyer SL, et al. Homozygous mutations in MFN2 cause multiple symmetric lipomatosis associated with neuropathy. Hum Mol Genet. 2015;24(18):5109-5114.

22. Santel A, Frank S, Gaume B, Herrler M, Youle RJ, Fuller MT. Mitofusin-1 protein is a generally expressed mediator of mitochondrial fusion in mammalian cells. J Cell Sci. 2003; 116(Pt 13):2763-2774.

23. Chen H, McCaffery JM, Chan DC. Mitochondrial fusion protects against neurodegeneration in the cerebellum. Cell. 2007;130(3):548-562.

24. Pham AH, Meng S, Chu QN, Chan DC. Loss of $\mathrm{Mfn} 2$ results in progressive, retrograde degeneration of dopaminergic neurons in the nigrostriatal circuit. Hum Mol Genet. 2012;21(22):4817-4826.

25. Papanicolaou KN, et al. Mitofusins 1 and 2 are essential for postnatal metabolic remodeling in heart. Circ Res. 2012;111(8):1012-1026.

26. Misko AL, Sasaki Y, Tuck E, Milbrandt J, Baloh RH. Mitofusin 2 mutations disrupt axonal mitochondrial positioning and promote axon degeneration. J Neurosci. 2012;32(12):4145-4155.

27. Detmer SA, Chan DC. Complementation between mouse Mfn1 and Mfn2 protects mitochondrial fusion defects caused by CMT2A dis- ease mutations. JCell Biol. 2007;176(4):405-414. 28. Detmer SA, Vande Velde C, Cleveland DW, Chan DC. Hindlimb gait defects due to motor axon loss and reduced distal muscles in a transgenic mouse model of Charcot-Marie-Tooth type 2A. Hum Mol Genet. 2008;17(3):367-375.

29. Cartoni R, et al. Expression of mitofusin 2(R94Q) in a transgenic mouse leads to CharcotMarie-Tooth neuropathy type 2A. Brain. 2010;133(Pt 5):1460-1469.

30. Strickland AV, et al. Characterization of the mitofusin 2 R94W mutation in a knock-in mouse model. J Peripher Nerv Syst. 2014;19(2):152-164.

31. Bannerman P, Burns T, Xu J, Miers L, Pleasure D. Mice hemizygous for a pathogenic mitofusin-2 allele exhibit hind limb/foot gait deficits and phenotypic perturbations in nerve and muscle. PLoS ONE. 2016;11(12):e0167573.

32. Baloh RH, Schmidt RE, Pestronk A, Milbrandt J. Altered axonal mitochondrial transport in the pathogenesis of Charcot-Marie-Tooth disease from mitofusin 2 mutations. J Neurosci. 2007;27(2):422-430.

33. Klein CJ, et al. Large kindred evaluation of mitofusin 2 novel mutation, extremes of neurologic presentations, and preserved nerve mitochondria. Arch Neurol. 2011;68(10):1295-1302.

34. Tufano M, et al. Early onset Charcot-Marie-Tooth neuropathy type $2 \mathrm{~A}$ and severe developmental delay: expanding the clinical phenotype of MFN2-related neuropathy. J Peripher Nerv Syst. 2015;20(4):415-418.

35. Ando $\mathrm{M}$, et al. Clinical and genetic diversities of Charcot-Marie-Tooth disease with MFN2 mutations in a large case study. J Peripher Nerv Syst. 2017;22(3):191-199.

36. Guyenet SJ, et al. A simple composite phenotype scoring system for evaluating mouse models of cerebellar ataxia. J Vis Exp. 2010;(39):e1787.

37. Seburn KL, Nangle LA, Cox GA, Schimmel P, Burgess RW. An active dominant mutation of glycyl-tRNA synthetase causes neuropathy in a Charcot-Marie-Tooth 2D mouse model. Neuron. 2006;51(6):715-726.

38. Lee J, et al. Overexpression of mutant HSP27 causes axonal neuropathy in mice. J Biomed Sci. 2015;22:43.

39. Misgeld T, Kerschensteiner M, Bareyre FM, Burgess RW, Lichtman JW. Imaging axonal transport of mitochondria in vivo. Nat Methods. 2007;4(7):559-561.

40. Verhoeven K, et al. MFN2 mutation distribution and genotype/phenotype correlation in Charcot-Marie-Tooth type 2. Brain. 2006;129(Pt 8):2093-2102.

41. Vallat JM, et al. Histopathological findings in hereditary motor and sensory neuropathy of axonal type with onset in early childhood associated with mitofusin 2 mutations. J Neuropathol Exp Neurol. 2008;67(11):1097-1102.

42. Santel A, Fuller MT. Control of mitochondrial morphology by a human mitofusin. JCell Sci. 2001;114(Pt 5):867-874.

43. Franco A, et al. Correcting mitochondrial fusion by manipulating mitofusin conformations. Nature. 2016;540(7631):74-79.

44. Cartoni R, Martinou JC. Role of mitofusin 2 mutations in the physiopathology of Charcot-Marie-Tooth disease type 2A. Exp Neurol.
2009;218(2):268-273.

45. Koshiba T, Detmer SA, Kaiser JT, Chen H, McCaffery JM, Chan DC. Structural basis of mitochondrial tethering by mitofusin complexes. Science. 2004;305(5685):858-862.

46. Cao YL, et al. MFN1 structures reveal nucleotide-triggered dimerization critical for mitochondrial fusion. Nature. 2017;542(7641):372-376.

47. Mattie S, Riemer J, Wideman JG, McBride HM. A new mitofusin topology places the redox-regulated $\mathrm{C}$ terminus in the mitochondrial intermembrane space. J Cell Biol. 2018;217(2):507-515.

48. Yan L, et al. Structural basis for GTP hydrolysis and conformational change of MFN1 in mediating membrane fusion. Nat Struct Mol Biol. 2018;25(3):233-243.

49. Brockmann K, et al. Cerebral involvement in axonal Charcot-Marie-Tooth neuropathy caused by mitofusin2 mutations. J Neurol. 2008;255(7):1049-1058.

50. Feely SM, et al. MFN2 mutations cause severe phenotypes in most patients with CMT2A. Neurology. 2011;76(20):1690-1696.

51. Stuppia G, et al. MFN2-related neuropathies: Clinical features, molecular pathogenesis and therapeutic perspectives. J Neurol Sci. 2015;356(1-2):7-18.

52. Bernard-Marissal N, et al. Altered interplay between endoplasmic reticulum mitochondria in Charcot-Marie-Tooth type 2A neuropathy [published online ahead of print, January 18, 2019]. Proc Natl Acad Sci U S A. https://doi.org/10.1073/ pnas.1810932116

53. Geisler S, et al. PINK1/Parkin-mediated mitophagy is dependent on VDAC1 and p62/SQSTM1. Nat Cell Biol. 2010;12(2):119-131.

54. Rocha AG, et al. MFN2 agonists reverse mitochondrial defects in preclinical models of Charcot-Marie-Tooth disease type 2A. Science. 2018;360(6386):336-341.

55. Shutt T, Geoffrion M, Milne R, McBride HM The intracellular redox state is a core determinant of mitochondrial fusion. EMBO Rep. 2012;13(10):909-915.

56. Wong YC, Ysselstein D, Krainc D. Mitochondria-lysosome contacts regulate mitochondrial fission via RAB7 GTP hydrolysis. Nature. 2018;554(7692):382-386.

57. El Fissi N, et al. Mitofusin gain and loss of function drive pathogenesis in Drosophila models of CMT2A neuropathy. EMBO Rep. 2018;19(8):e45241.

58. Bergamin G, Cieri D, Vazza G, Argenton F, Mostacciuolo ML. Zebrafish Tg(hb9:MTS-Kaede): a new in vivo tool for studying the axonal movement of mitochondria. Biochim Biophys Acta. 2016;1860(6):1247-1255.

59. Brownlees J, et al. Charcot-Marie-Tooth disease neurofilament mutations disrupt neurofilament assembly and axonal transport. Hum Mol Genet. 2002;11(23):2837-2844.

60. d'Ydewalle C, et al. HDAC6 inhibitors reverse axonal loss in a mouse model of mutant HSPB1-induced Charcot-Marie-Tooth disease. Nat Med. 2011;17(8):968-974

61. Gentil BJ, Minotti S, Beange M, Baloh RH, Julien JP, Durham HD. Normal role of the low-molecular-weight neurofilament protein 
in mitochondrial dynamics and disruption in Charcot-Marie-Tooth disease. FASEB J. 2012;26(3):1194-1203.

62. Kalmar B, et al. Mitochondrial deficits and abnormal mitochondrial retrograde axonal transport play a role in the pathogenesis of mutant Hsp27-induced Charcot Marie Tooth Disease. Hum Mol Genet. 2017;26(17):3313-3326.

63. Knott AB, Perkins G, Schwarzenbacher R, Bossy-Wetzel E. Mitochondrial fragmentation in neurodegeneration. Nat Rev Neurosci.
2008;9(7):505-518.

64. Wang L, et al. Mitofusin 2 Regulates axonal transport of calpastatin to prevent neuromuscular synaptic elimination in skeletal muscles. Cell Metab. 2018;28(3):400-414.e8

65. Caroni P. Overexpression of growth-associated proteins in the neurons of adult transgenic mice. JNeurosci Methods. 1997;71(1):3-9.

66. Tsai Y, et al. Human iPSC-derived neural progenitors preserve vision in an AMD-like model. Stem Cells. 2015;33(8):2537-2549.
67. Wang S, et al. Non-invasive stem cell therapy in a rat model for retinal degeneration and vascular pathology. PLOS ONE. 2010;5(2):e9200.

68. Bogdanik LP, et al. Loss of the E3 ubiquitin ligase LRSAM1 sensitizes peripheral axons to degeneration in a mouse model of Charcot-Marie-Tooth disease. Dis Model Mech. 2013;6(3):780-792.

69. Ho R, et al. ALS disrupts spinal motor neuron maturation and aging pathways within gene co-expression networks. Nat Neurosci. 2016;19(9):1256-1267. 